\title{
Investigation into the turbulence statistics of installed jets using hot-wire anemometry
}

\author{
Anderson Proença ${ }^{1}$ D $\cdot$ Jack Lawrence ${ }^{1} \cdot$ Rod Self $^{1}$
}

Received: 16 May 2020 / Revised: 18 July 2020 / Accepted: 12 September 2020 / Published online: 1 October 2020

(c) The Author(s) 2020

\begin{abstract}
This work presents a detailed study of the turbulence flow statistics of a jet mounted with its axis parallel to a rigid flat plate. Hot-wire constant temperature anemometry has been used to measure the single-point and two-point statistics of the axial velocity component at several locations within the jet flow field. Results show that the jet mean flow near the plate surface is subjected to a local acceleration and redirection due to a Coandă-type effect. The propagation of these effects downstream of the plate trailing edge is strongly dependent on the plate position. Regarding the velocity fluctuations, the mean turbulence intensity levels are seen to decrease as the radial distance between the jet and surface decreases. Analysis of the single-point power spectral density data on the shear layer close to the plate shows that the reduction in magnitude of the low-frequency content of the energy spectrum is responsible for the decrease in turbulence intensity. Additionally, the characteristic time and length scales computed from two-point measurements reduce as the plate is mounted closer to the jet centre-line. The axial eddy convection velocity is seen to increase in the region of high turbulent kinetic energy in the shear layer adjacent to the surface. Empirical models for turbulence characteristic scales and eddy convection velocity are presented. These findings suggest that both the amplitude and distribution of the jet mixing noise sources are affected when closely installed next to a surface. This paper is a continuation of a recent investigation on the turbulence statistics of isolated jets presented in Proença (Exp Fluids 60(4):63, 2019).
\end{abstract}

\section{Graphic abstract}

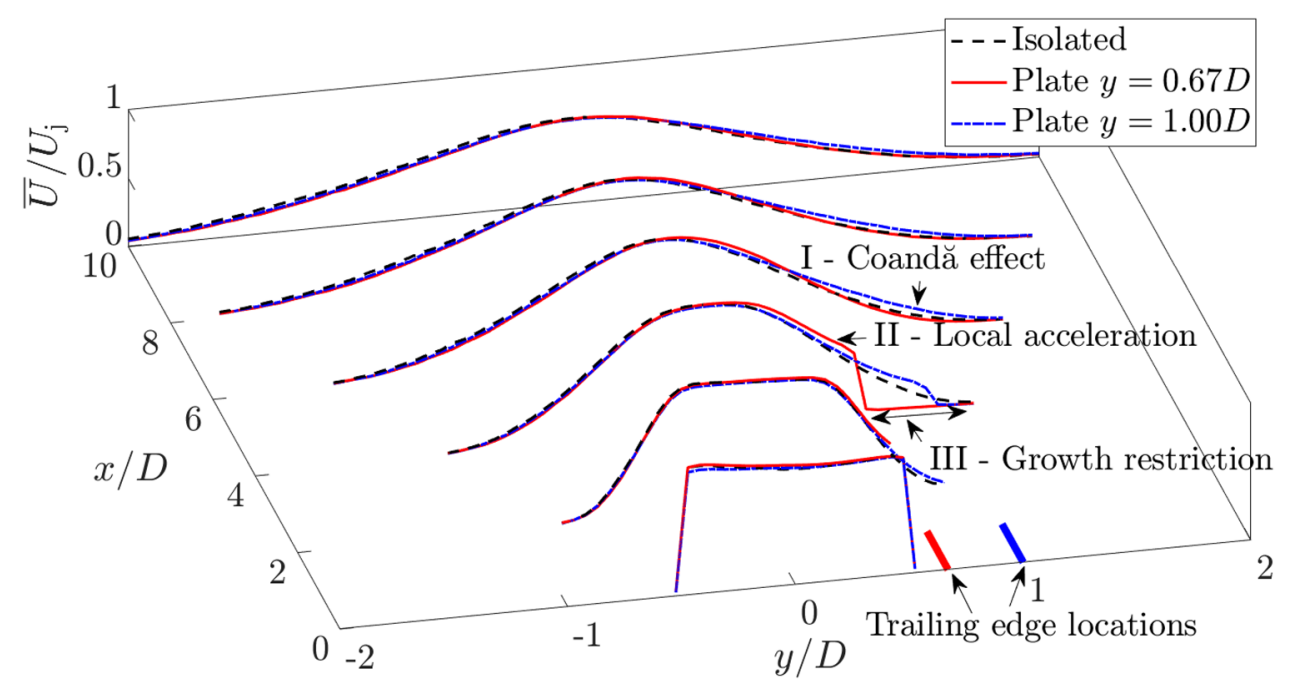

Anderson Proença

a.proenca@soton.ac.uk

1 Institute of Sound and Vibration Research (ISVR), University of Southampton, Southampton SO17 1BJ, UK 


\section{Introduction}

Modern ultra-high-bypass ratio (UHBR) turbofans are expected to be installed in a close-coupled position underneath the wings of commercial aircraft. In these configurations, the exhausted jet will likely interact ostensibly with wing and high-lift devices. When a jet interacts with a solid boundary, both the flow field and the hydrodynamic pressure field will be somewhat modified. Two main questions, therefore, arise: (1) by how much are these two fields modified, and (2) what is the impact on aerodynamic performance and noise generation? Researchers in industry and academia have been investigating this topic to mitigate issues related to the reduction in aerodynamic efficiency and noise augmentation.

Jet-surface interaction effects are present in a broad range of applications. Impinging jets have been studied extensively for short take-off and landing (STOL) systems (Korbacher 1974; Ho and Nosseir 1981; Nosseir and Ho 1982) and cooling systems for turbine vanes of blades (Gauntner et al. 1970) are just two examples. Research has also been conducted on water-installed jets (Madnia and Bernal 1994; Tian et al. 2012; Roy et al. 2018), showing significant changes in the jet mean flow and turbulence intensity. These surveys, however, are usually limited to very low Reynolds numbers and focused on regions far away from the main noise-producing regions of the jet (i.e. $x / D>30$ ).

Recently, research has been carried out on installed jets based on realistic jet-wing geometries. Most efforts have been directed at detailed investigations of the far-field pressure from installed jet configurations (Lawrence et al. 2011; Brown 2013; Cavalieri et al. 2014; Khavaran 2015; Zaman et al. 2015; Faranosov et al. 2019; Meloni et al. 2020). Jet installation effects for relatively simple flat plate surface geometries and single stream jet flows are reasonably well understood and various jet-surface interaction noise models have been successfully developed (Lyu et al. 2017; Vera 2018; Bychkov and Faranosov 2018; Dawson et al. 2020). However, these models incorporate three key assumptions: (1) the jet remains axisymmetric below the surface, (2) the surface is located outside of the jet's rotational hydrodynamic field, and (3) the jet turbulent field is not modified by the surface. These hypotheses are likely not valid for particularly closely coupled configurations (i.e. when the vertical separation between jet and surface trailing edge is small). Furthermore, following Lighthill's pioneering discovery (Lighthill 1952, 1954), it is well known that jet mixing noise is proportional to the volume integral of the Reynolds stresses within the jet and, thus, any changes to the turbulence statistics of installed jets should be included in any acoustic analogy-based noise prediction methodologies.

In comparison to the acoustics, research on the aerodynamics of installed jets is much more limited.
Investigations into the mean flow development of a jet beneath an infinitely long flat plate show that the turbulence statistics are strongly modified in locations where the plate restricts the growth of the jet shear layer (Di Marco et al. 2013; Mancinelli et al. 2017). Similar results for finite plate configurations have also been published (Smith and Miller 2013; Brown and Wernet 2014; Cavalieri et al. 2014); however, most of the plate locations are unrepresentative of future UHBR geometries. Finally, as far as the authors are aware, no exhaustive description of the two-point statistics has been attempted thus far.

In this work, a simplified version of the complete installed jet problem is studied via a small-scale experiment. A single-stream, unheated jet is mounted adjacent to a flat plate at a range of locations representative of full-scale aircraft and several jet Mach numbers are studied ( $M=0.2$ to 0.8 ). The trailing edge of the plate is positioned at four locations, all chosen based on standard configurations used in recent industrial research projects (JERONIMO, HARMONY). The unsteady velocity field was measured using hot-wire anemometry across several planes of the jet. Single-point and two-point measurements were performed. The flat plate induces changes in the mean velocity, the mean turbulence intensity and the characteristic turbulence length- and time scales. Results presented here extend findings recently published in Proença et al. (2019) and Proença et al. (2020), where only isolated jets were considered.

This paper is structured as follows. In Sect. 2, key information about the facility, equipment, data processing and parameter definitions are presented. Results are then examined in the three subsequent sections. First, in Sect. 3, changes in the statistical central moments of the unsteady velocity field due to the presence of the plate are investigated. Then, in Sect. 4, two-point statistical data are used to study the characteristic scales of the isolated and installed jet turbulence both in the time and frequency domains. Finally, in Sect. 5, a discussion concerning viscous effects near the plate surface region is presented.

\section{Experimental methodology}

\subsection{Experimental facility and experimental hardware}

Experiments were performed in the ISVR Doak Laboratory at the University of Southampton, UK. The facility has dimensions of, approximately, $15-\mathrm{m}$ long by $7-\mathrm{m}$ wide by 5 -m high. The air jet is supplied by a high-pressure compressor-reservoir system. A labyrinth plenum is located inside the anechoic chamber, upstream of the jet nozzle exit. The flow then leaves the facility passively via a collector located on the wall opposite to the jet rig. The jet rig is capable 


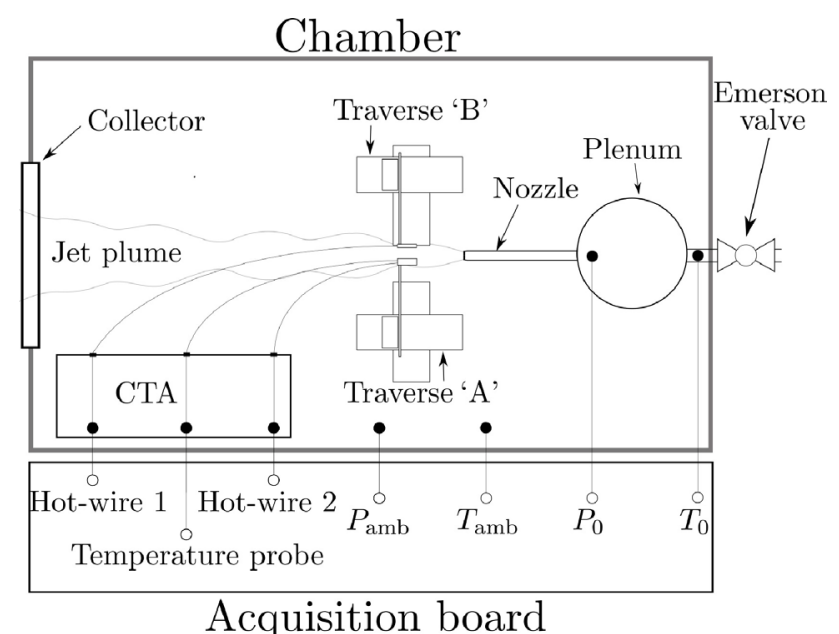

(a)

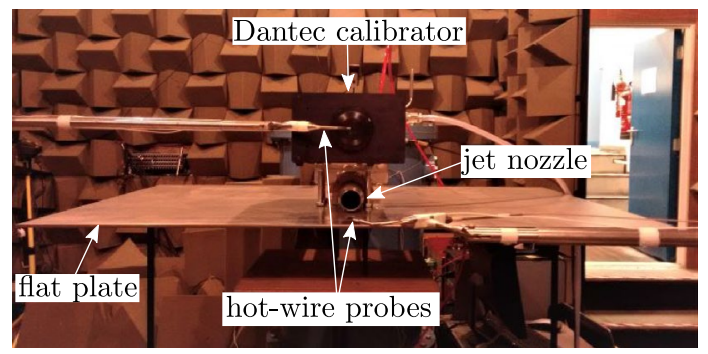

(b)

Fig. 1 a Schematic showing the Doak Laboratory static jet rig and sensors; $\mathbf{b}$ image of the jet-plate configuration during hot-wire calibration

of achieving a controlled exit Mach number ranging from $M=0.15$ up to $M=1$. Single-stream jet tests can, therefore, be carried out on flow regimes characteristic of civil aircraft. The key features of the laboratory and an image of the static jet rig are illustrated in Fig. 1.

The jet nozzle used in this study is a 38.1-mm-diameter, convergent nozzle with a relatively high convergence angle of $14^{\circ}$. The acoustics and aerodynamics, as well as information about the nozzle geometry of the jet, have been published in the previous work Lawrence (2014); Proença (2018). As the bypass ratio of modern aircraft engines increases, the larger secondary unheated mass flow is expected to dominate the jet-wing interaction physics such that one can neglect the hot core flow. Thus, single-stream unheated jets are capable of representing the pertinent physics of the full-scale problem.

Installation effects were studied by mounting a flat plate in the vicinity of the jet plume. The flat plate used has a span of $1.1 \mathrm{~m}$ (i.e. over 14 jet diameters either side of the jet centre-line) and chord of $0.762 \mathrm{~m}$ (i.e. 20 jet diameters). The plate was made of 6.4-mm-thick aluminium. Two additional bars of aluminium were welded along the span-wise length of the wing to ensure rigidity, especially at the trailing edge.
The plate was then attached to the jet pipe upstream of the nozzle exit.

To survey the aerodynamics, two ISEL traverse systems were mounted next to the jet. One of the traverses was configured with three motor power modules, allowing independent movement along the $x, y$ and $z$ planes. This 3 -axis traverse was used for the single-point measurements and for the moving probe in the two-point measurements. The second traverse system had two motor power modules for moving along the $x$ and $y$ planes. Any vertical $z$-plane movement was performed manually. This 2-axis traverse was used to support the fixed probe during the two-point measurement campaign. The unavoidable uncertainty arising from the alignment between the traverse system and jet nozzle alignment is one of the main source of error. Extra care was taken to find the optimal alignment of the system and all test points were measured with the same traverse reference origin, so errors are consistent between builds. Further information concerning the traverse systems and nozzle alignment procedure can be found in Proença (2018).

The ambient chamber properties were measured using an Omega HX94V probe, for temperature and relative humidity, and a Druck DPI-142 barometer. The total temperature and pressure of the flow upstream of the nozzle exit were measured in the plenum using an Omega K-type thermocouple and a Druck PDCR-820 1 bar transducer, respectively.

\subsection{Hot-wire anemometry}

A Dantec hot-wire constant temperature anemometry (CTA) system was used to measure the unsteady velocity field. The four main advantages to using CTA are: (1) high spatial resolution (compared to multi-hole probes) (2) low cost, (3) high frequency resolution (essential for small-scale experiments), ${ }^{1}$ and (4) fast post-processing (compared to optical techniques). The main disadvantages are probe interference effects and time due to the requirement to make point-wise measurements. Probe interference effects are only significant in high jet velocity regions, i.e. in the quasi-laminar potential core region (Proença et al. 2019), which is not of interest for installed jet research.

Single-miniature hot-wire probes (Dantec 55P11) were used in this experiment to measure the instantaneous resultant velocity field. It is common practice, however, to assume that the hot-wire measures the stream-wise component of the flow when the probe stem axis is aligned with the jet axis (Laurence 1956; Davies et al. 1963; Bruun 1995; HarperBourne 1999, 2003; Morris and Zaman 2010; Proença et al.

\footnotetext{
${ }^{1}$ Strouhal numbers up to 10 are of interest, or frequencies below 70 $\mathrm{kHz}$ for the $1 / 50$ th-scale model used in this work
} 
2019). Thus, all the data presented herein refer to the axial component of the velocity field.

The hot-wire probes were calibrated in situ using a Dantec StreamLine Manual Calibrator over the range of velocities of interest (i.e. $5-300 \mathrm{~m} / \mathrm{s}$ ). The calibration coefficients were extracted from a 4th-order polynomial curve-fit. A temperature probe was mounted near the hot-wire sensor to account for temperature fluctuations present during both calibration and test point measurements. The estimated relative expanded uncertainty in a velocity sample is within $\pm 2.5 \%$ (see Wang et al. (2020) for more details).

The voltage fluctuation measured by a CTA hot-wire system is associated with the fluctuations in momentum flux, $\rho u$. Providing that the local density fluctuations are small in comparison to the velocity fluctuations, compressibility effects are negligible and the calibration curve converts voltage data to velocity. This is particularly true for low and moderate subsonic air jets as: (1) the velocity fluctuations dominate the density fluctuations by at least one order of magnitude, and (2) the structure of the shear layer turbulence is unaltered up to sonic Mach numbers (Bradshaw 1977). Additionally, most measurements carried out in the present work were performed in the jet shear layer, particularly along the lipline, where $\bar{U} \leq 0.6 U_{\mathrm{j}}$ (maximum local Mach number $M=0.36$ ). Both the single- and two-point statistics of the isolated jet turbulent velocity field studied here are seen to collapse at least up to $M=0.8$ (Proença 2018).

\subsection{Data acquisition and post-processing}

Data were acquired using a 24-bit National Instruments dynamic signal acquisition system. An eight-channel NI PXI-4472 was used to acquire ambient chamber and flow data. These signals were sampled at $1 \mathrm{kHz}$ and used to compute the nominal jet and flight exit velocities.

Hot-wire measurements were recorded using a sampling rate of $50 \mathrm{kHz}$. All data test points were acquired for a total time of ten seconds. The hot-wire voltage signal was corrected for temperature fluctuations during measurements as described by the manufacturer (Jørgensen 2002). The corrected voltage was then converted to velocity using the polynomial curve-fit obtained in the calibration procedure. Statistical central moments up to the fourth-order were then calculated and stored in matrices.

The power spectral density (PSD) was calculated using Welch's method. The velocity time series was divided into segments, each segment containing 4096 samples. A 50\% overlap between segments and a Hanning window were used. The fast Fourier transform (FFT) of each segment was then calculated and averaged. The PSD was then computed by the product of the averaged FFT multiplied by its complex conjugate. The cross power spectral density (CPSD) was computed similarly, except the averaged FFT calculated

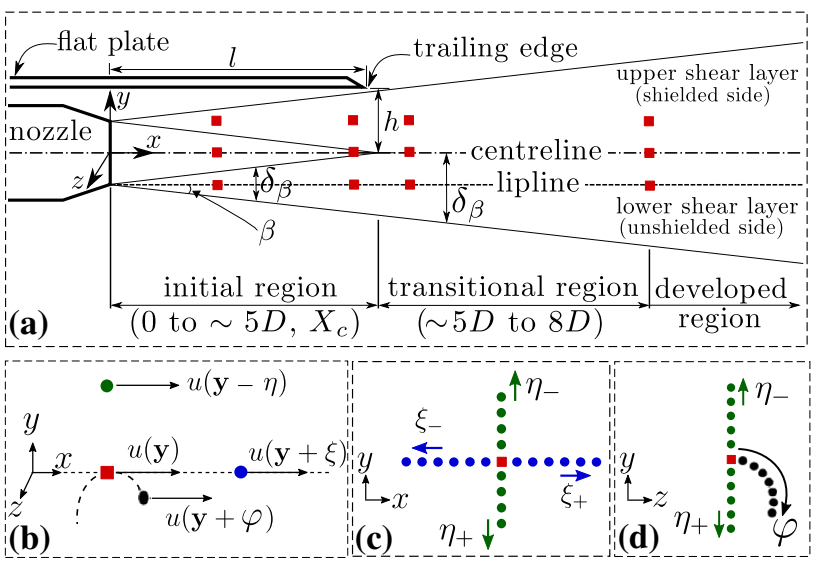

Fig. 2 Coordinate systems and parameters used in this work: a jet regions and jet-plate configuration; b schematic of the separation vector relative to the reference probe location $\zeta(\xi, \eta, \varphi)$; c separation vector in the $x$ - $y$ plane; and $\mathbf{d}$ separation vector in the $y$ - $z$ plane. Square symbols represent reference probe locations for the two-point measurements. Circles illustrate some positions of the moving sensor along the separation axes

Table 1 Definition of plate configurations investigated in this work

\begin{tabular}{llllll}
\hline Build & 'B0' & 'B1' & 'B2' & 'B3' & 'B4' \\
\hline$h / D$ & - & 0.67 & 1.00 & 0.67 & 1.00 \\
$l / D$ & - & 4.00 & 4.00 & 2.00 & 2.00 \\
\hline
\end{tabular}

for the first probe was multiplied by the complex conjugate of the FFT obtained by the second probe. Finally, correlation functions were obtained by applying the inverse FFT to the PSD and CPSD results.

\subsection{Coordinate system and test matrix}

The origin of the coordinate system used in this work is located at the centre of the jet nozzle exit. The shear layer above the centre-line of the jet (i.e. $y>0$ ) is henceforth referred to as the 'upper shear layer' or 'shielded side'. Locations where $y<0$ are referred to in the text as the 'lower shear layer' or 'unshielded side'. Figure 2 displays the coordinate system and the key parameters used to describe the jet-plate configuration.

During a preliminary test, in addition to the isolated jet case (i.e. Build 0), four installed jet-plate 'height', $h$, and plate 'length', $l$, combinations were investigated, as shown in Table 1 . This preliminary study suggested that the velocity fields of the configurations with the shortest plate lengths (i.e. Builds 3 and 4) did not differ significantly from the isolated jet case (Proenç et al. 2017). This suggests that the plate did not restrict the development of the jet. In the final test campaign, therefore, the majority of measurements were 
performed for Builds 0,1 and 2 to understand if and how the jet development had been altered.

Single-point hot-wire measurements were performed from the nozzle exit down to $x / D=15$, at one jet diameter intervals. Radial mean velocity profiles were obtained along both the $y$-axis and the $z$-axis. Additional radial mean velocity profiles were acquired in locations close to the plate surface in an attempt to describe the boundary layer generated on the plate due to the jet. Two-point hot-wire measurements were performed in the locations illustrated in Fig. 2 (i.e. with the reference sensor located at $x / D=2,4,5,8 ; y / D=0.5$, 0.0 , and -0.5 ). For each reference sensor location, the moving sensor was positioned at 8 points along the $y$-axis and 12 points along the $x$-axis, as shown in Fig. 2c, d. The 12 reference sensor locations defined above were chosen based on recently published experimental (Proença et al. 2019) and large eddy simulation (Wang et al. 2020) works. For subsonic isolated jets, the turbulence statistics are seen to collapse both within the nominally laminar potential core and the transitional region of the shear layer. Measurements were carried out at the following Mach numbers: $M=0.2$, $0.4,0.6,0.8$, except for the two-point test where it was not possible to achieve robust data at $M=0.8$ due to a significant amount of probe vibration, often resulting in hot-wire damage.

\section{Results: statistical moments}

First, results of the single-point statistics are presented and discussed. The first four central moments are examined in the next three subsections. For particular configurations, the statistical moments indicate significant changes to the velocity and turbulence structure of the jet due to the presence of the plate. To explain the physical flow structure behind the change in turbulence level, the PSD spectra are also presented. Finally, higher-order statistics (i.e. skewness and kurtosis) are used to study the degree of flow non-uniformity and intermittency near the plate surface.

\subsection{Jet mean velocity}

Due to the high convergence angle of the nozzle used, the spreading rate of the isolated jet is relatively high, $\beta=0.12$ $\mathrm{rad}$ (Proença et al. 2019). This angle suggests that the jet plume impacts on the plate surface at $x / D=1.41$ in Build 1. The jet would 'just miss' the plate trailing edge in Build 2 , as the edge of the shear layer for the radial distance $r=D$ is at the axial location $x / D=4.15$ (as illustrated in Fig. 2a). However, the presence of the plate causes two effects on the jet mean flow that change that picture, that is, 1) a jet local acceleration and 2) a jet redirection.
To further discuss these effects, key mean velocity profiles are shown in Fig. 3. Data for the isolated and installed cases are displayed. The local mean velocity $\bar{U}$ is normalised by the maximum velocity measured along the jet centre-line, $U_{j}$. The nominal jet Mach number for all profiles illustrated in this figure is $M=0.6$. Similar trends were observed for the other jet velocities studied. The installed cases displayed are Builds 1 and 2 as defined in Table 1.

In Fig. 3a, a small difference in the jet mean velocity values is seen in the upper shear layer of installed configurations compared to the isolated case. Data measured upstream of the axial location $x / D=2$ (e.g. $x / D=0,1$, and 1.5) were seen to produce similar curves for the different configurations. Thus, although not directly measured, the jet is expected to wet the plate at $x \approx 1.4 D$ in Build 1 , which is the location calculated from the jet spreading rate.

Further downstream, the mean velocity is consistently higher in the upper shear layer of installed configurations. This is illustrated in Fig. 3b, where the profiles are measured at $x / D=4$. The following three main conclusions can be drawn from that sub-figure: (1) the local mean flow accelerates significantly in Build 1 (red curve) at locations $y>0$; (2) the jet mean flow actually interacts ostensibly with the plate in locations close to the trailing edge of Build 2 (blue curve), and (3) isolated and installed jet mean velocity values collapse well in the shear layer opposite to the plate.

Two different physical mechanisms are responsible for claims 1 and 2 mentioned above. In Build 1, the plate restricts the development of the jet from $x=1.4 D$. Momentum in the direction perpendicular to the plate is transferred to the flow momentum in the spanwise and longitudinal directions. This local acceleration effect produces the 'skewed' profile of Build 1 seen in Fig. 3b, resembling a wall jet flow development (Gupta et al. 2020). Mean velocities near the trailing edge of the plate are as much as $10 \%$ higher in Build 1 compared to an isolated jet.

For Build 2, a local acceleration is also seen in locations close to $y=D$, a narrower region in comparison to this effect on Build 1 . The mean velocity profile indicates that the mean flow impacted on the plate surface in a location upstream of the plate trailing edge. This was not expected from the isolated jet spreading rate. Therefore, an extra mechanism must have acted upon the jet, redirecting the mean flow towards the plate. An explanation for this phenomenon is the change in the entrainment process caused by the presence of the plate. The plate restricts the amount of flow entrainment causing the jet to move towards the platea behaviour referred to as the Coandă effect (Tritton 1988).

Analysis of Fig. 3c, d shows that the local acceleration effects seen on Build 1 reduces in locations downstream of 


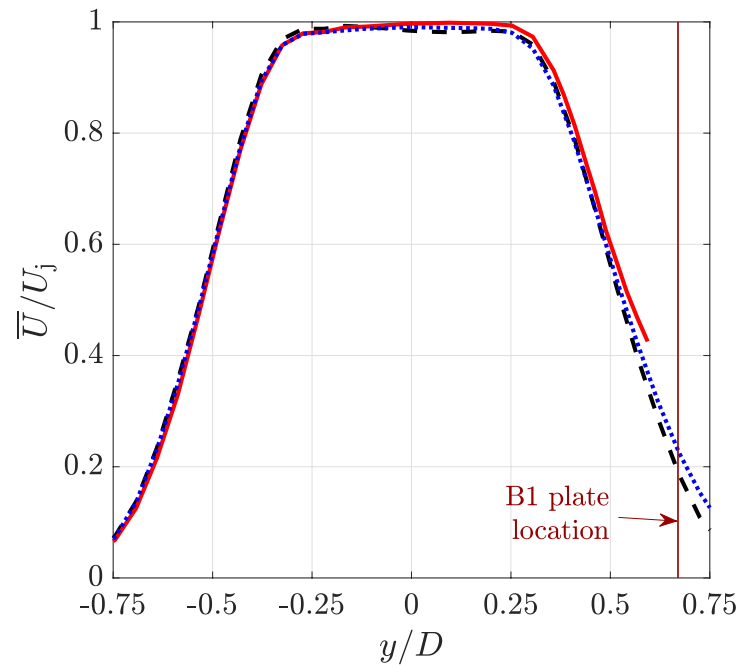

(a) $x / D=2$

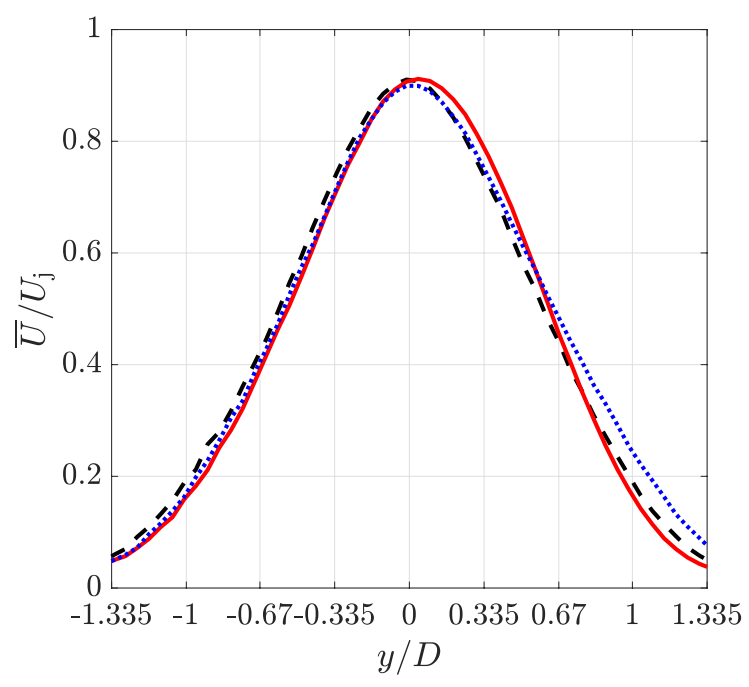

(c) $x / D=6$

Fig. 3 Jet mean velocity profiles measured for Builds 0,1 and 2 . The profiles shown were measured are at $\mathbf{a} x / D=0 ; \mathbf{b} x / D=4 ; \mathbf{c}$ $x / D=6$; and d) $x / D=10$. Black, dashed lines for the isolated jet

the plate trailing edge. Redirection effects on Build 2 are clearly identifiable at $x / D=10$, but the maximum difference in comparison to the isolated case is not more than $3 \%$ of the jet exit velocity. It is important to stress that the uncertainty of the present experiments is $\pm 1.5 \%$ on the velocity sample (as discussed in Sect. 2.2).

For the shorter plate length builds (i.e. Builds 3 and 4 with $l=2 D$ ), the mean velocity data are seen to match that of the isolated jet case. In summary, the mean axial velocity of a jet is modified by a plate either when the flow impacts the plate surface, creating a wall jet-type profile, or when the surface is sufficiently close to the jet, thus changing the jet entrainment and inducing a Coandă effect. In the following subsections, higher-order statistical

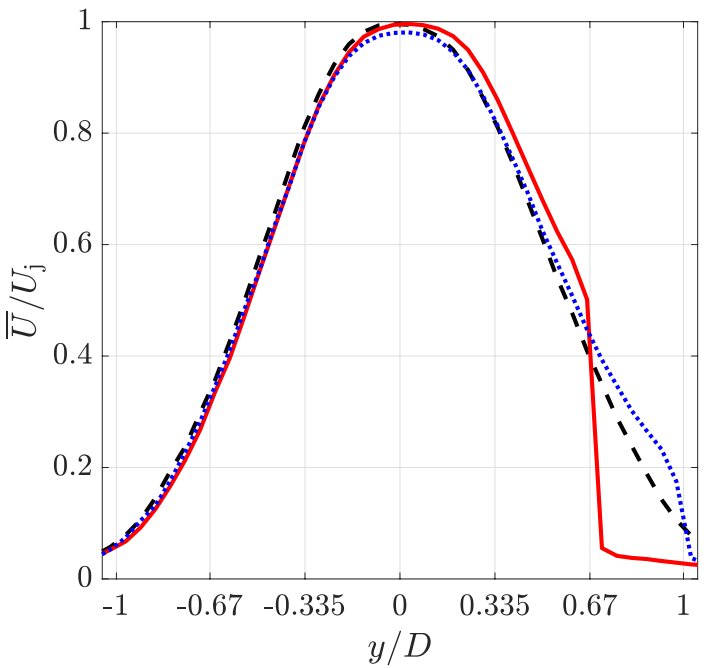

(b) $x / D=4$

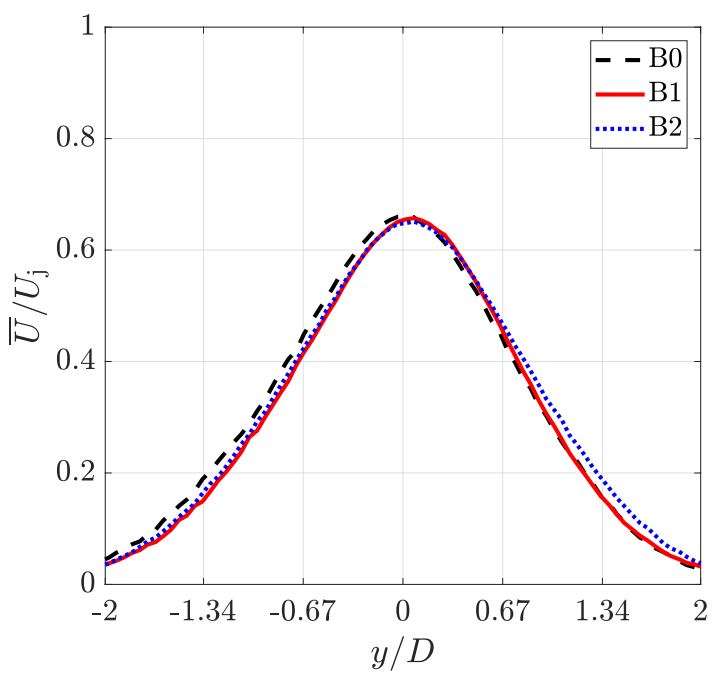

(d) $x / D=10$

(B0); red, solid lines for the $h=0.67 D, l=4 D$ installed jet (B1); blue, dotted lines for the $h=D, l=4 D$ installed jet (B2). Nominal jet Mach number $M=0.6$

moments are analysed to investigate the changes to the structure of the turbulence.

\subsection{Jet turbulence levels}

In comparison to the isolated case, the turbulence levels of installed jets are consistently seen to decay in the upper shear layer, principally close to the plate surface. Figure 4 displays the turbulence levels of Builds 0,1 and 2 measured along two axial locations (Fig. 4a) and along the centreline and lipline (Fig. 4b). Note that the turbulence intensity (TI) profile is consistently skewed at the jet nozzle exit. This asymmetry is believed to be the result of a small misalignment between the traverse system and the jet nozzle. The 


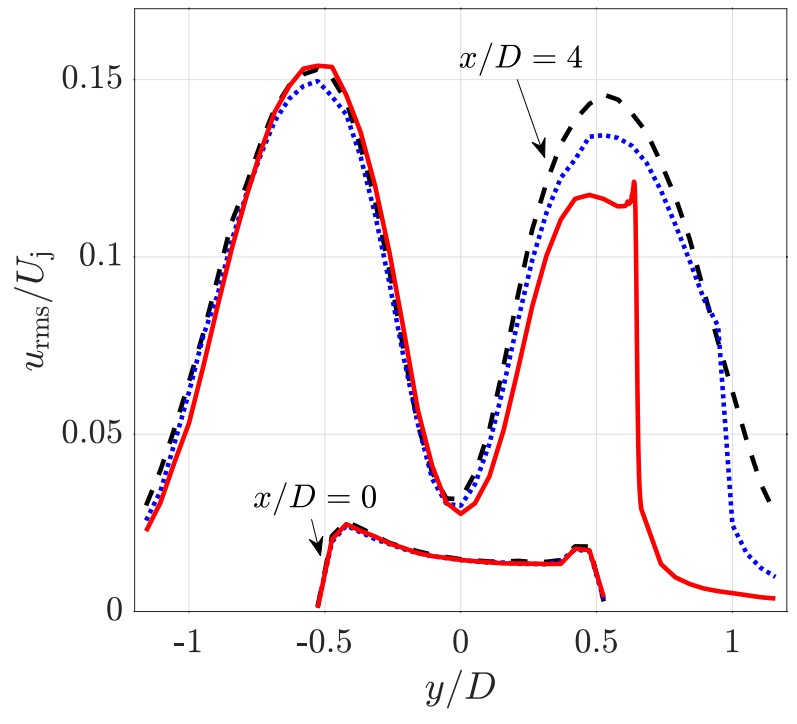

(a) Turbulence intensity radial profiles

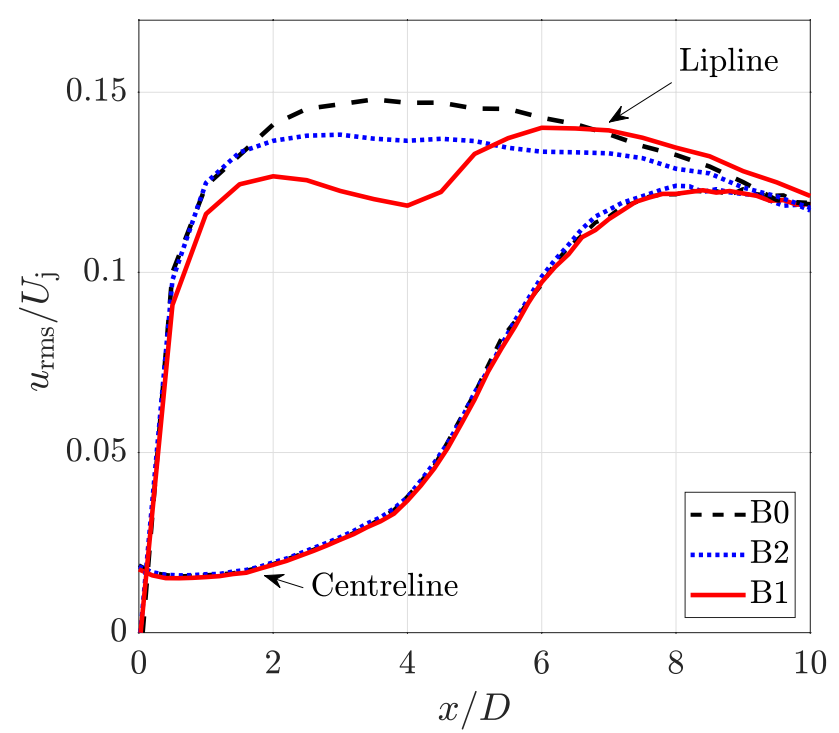

(b) Turbulence intensity axial profiles

Fig. 4 Jet turbulence intensity. a Radial profiles at the nozzle exit and at $x / D=4$. b Axial profiles along the jet centreline and jet lipline. Black, dashed lines for isolated jets (B0); red, solid lines for installed $h / D=0.67, l / D=4$ jet (B1); blue, dotted lines for installed $h / D=1.00, l / D=4$ jet (B2). Nominal jet Mach number $M=0.6$

difference in peak TI level either side of the jet centre-line, however, does not exceed $0.01 U_{\mathrm{j}}$. As discussed in Sect. 2.2, this difference is within the uncertainty bounds of the experiment.

Regarding the radial profiles shown in Fig. 4a, the turbulence intensity of all three configurations collapses well at the jet nozzle exit and decreases consistently at the shielded side as the plate moves closer to the jet. The turbulence intensity of the installed configurations tends to recover symmetry in locations further downstream. This is indicated by the data measured along the lipline illustrated at Fig. $4 \mathrm{~b}$. The variation of the turbulence intensity along the jet centreline due to the presence of the plate is negligible.

Additionally, immediately downstream of the trailing edge of the plate, a distinct rise in the turbulence intensity is seen. This is hypothesised as the detachment of the boundary layer developing on the surface of the plate. Note that this feature does not occur in the other installed configuration ( $h / D=1.00$, blue, dashed line). In configurations in which the jet flow field does not interact ostensibly with the solid boundary, the flow separation effect is expected to be weak and the turbulence levels do not rise significantly further downstream.

The local acceleration alone does not provide a fully explanation to the lower turbulence intensity in the upper shear layer in comparison to the lower shear layer. In fact, it implies the opposite: an increase in the local Reynolds number by the means of a higher local velocity would be responsible to an increase in the velocity fluctuations. Thus, the effect of the plate blockage on the development of the turbulence structures should also be addressed. The rigid surface interrupts the development of the large, coherent turbulence structures which are responsible for the injection of energy in the turbulence spectra. The eddy structure which grows with the shear layer width in isolated jets 'breaks down' and is confined by the surface in the installed configurations.

To exemplify this hypothesis, Fig. 5a, b shows radial profiles of the PSD of isolated and installed configurations for Strouhal numbers 0.1 and 1, respectively. To keep consistent with Fig. 4a, data in Fig. 5 were obtained at the trailing edge location, $x / D=4$. To put it simply, the lowfrequency content of the PSD resembles the results seen in the time domain, that is, the energy of large-scale structures decreases as the plate moves closer to the jet centreline. Less evident differences are seen at high-frequencies. However, at the location of the peak seen in Fig. 4a, a slight increasing trend is also seen for relatively high Strouhal numbers at $h=0.67 D$ in Fig. 5b. Although this is not definitive, it is a likely indication of the flow separation of a coherent structure with different scale of that found in the jet shear layer.

A possible second mechanism explaining the lower turbulence levels of installed jet configurations is the transfer of energy of the streamwise component of the velocity fluctuations to the transverse components of the velocity. This is believed to be secondary to the results presented above and further experiments or numerical simulations should be investigated to provide more information about this turbulence intensity behaviour.

The clear change of turbulence intensity in the shielded side of a installed jet configuration suggests that, for 


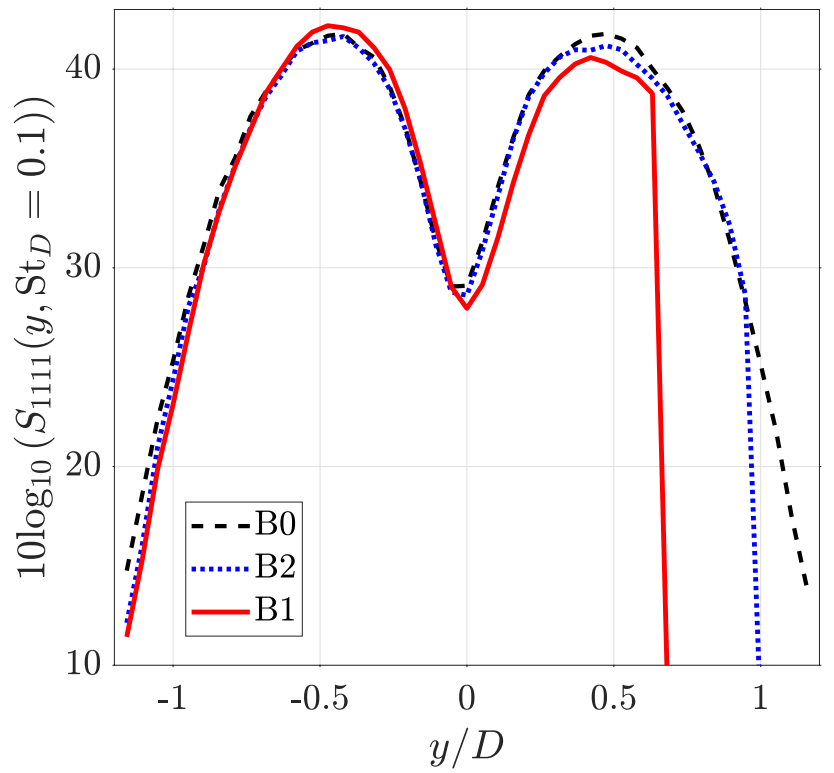

(a) $\mathrm{St}=0.1$

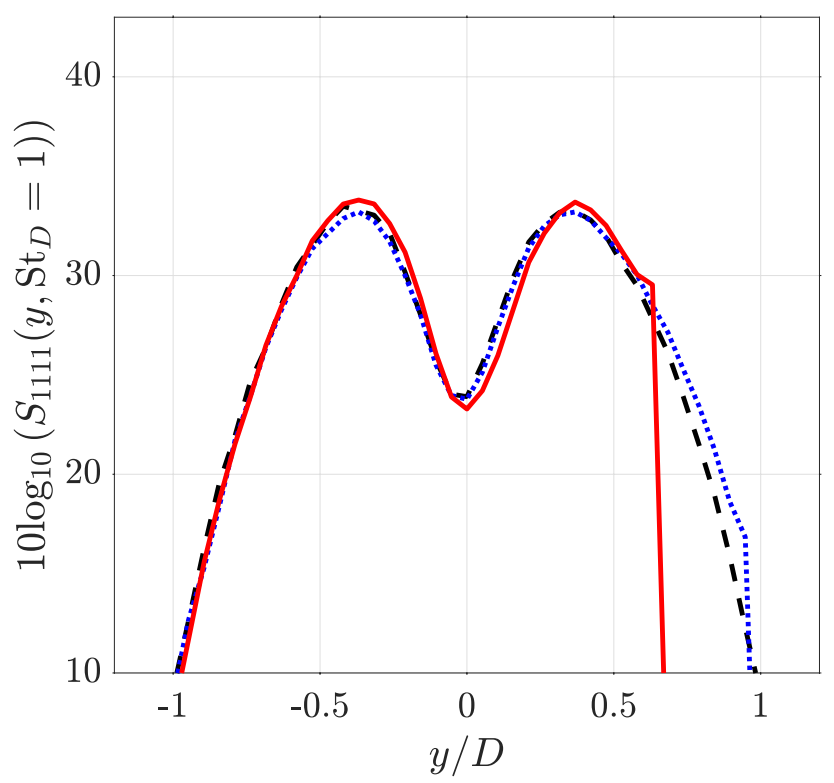

(b) $\mathrm{St}=1.0$

Fig. 5 Power spectral density of the square of the axial velocity fluctuations. Data displayed for Builds 0,1 and 2. $\mathbf{a ~ S t}=0.1$ and $\mathbf{b} \mathrm{St}=1$. Axial location $x / D=4$ and jet Mach number $M=0.6$

example, the jet mixing noise sources are altered. Thus, it is expected that understanding how a rigid surface (i.e. wing) alters the pure jet mixing noise in installed jet configurations is essential to predict full-scale jet noise accurately.

\subsection{High-order moments}

The importance of the higher-order moments in the present analysis is twofold. First of all, the fourth-order moment, kurtosis, is significant to jet noise modelling because it expresses information about the source strength and the accuracy of assumptions used to model the turbulent source region. For example, for a normal distribution, secondorder and fourth-order joint moments are simply related by a power of two (Monin and Yaglom 1975; Batchelor 1982). In subsonic jets, it then follows that a one-term exponential function can be used to model the cross-correlations in both the time and frequency domains.

Second, the third-order moment, skewness, describes the predominance of intermittent bursts of slow or fast moving structures. The skewness is, therefore, proportional to the difference between the eddy convection velocity and the local mean velocity (Fisher and Davies 1964). In subsonic jets, a quasi-normal distribution (skewness $\sim 0$ and kurtosis $\sim 3$ ) is seen in two regions: (1) in the fully developed turbulence region, many jet diameters downstream of the nozzle exit, and (2) in the maximum turbulence kinetic energy region (Harper-Bourne 2003; Morris and Zaman 2010; Proença et al. 2019), where the joint moments discussed in the next sections were measured.

Regarding the axial unsteady velocity of an isolated jet, a negative skewness is seen in the jet inner shear layer (i.e. $-0.5 \leq y / D \leq 0.5)$. This corresponds to more frequent highvelocity flow bursts and less frequent slow-velocity events and it is due to the strong presence of the nominally laminar, fast potential core. On the other hand, the skewness is positive in the outer shear layer. This is a direct effect of the mixing of the jet with the stationary medium, which generates predominantly slow flow speeds (Piquet 1999).

High-order moments of isolated and installed jets are displayed in Fig. 6. Several skewness and kurtosis radial profiles are shown in Fig. 6a, b, respectively. Figure 6c, d illustrate skewness and kurtosis measured at $x / D=4$. The data used in Fig. 6 are for an $M=0.6$ jet.

Within the inner shear layer, the high-order statistics of installed configurations present the behaviour expected for an isolated jet in most axial locations. The clear exception is on the trailing edge plane. As can be seen in Fig. 6c, d, slightly stronger intermittent events are expected in the inner shear layer of Build 1. One hypothesis to explain this trend is that the frequency of structures passing at the end of the jet potential core is increased due to the presence of the plate.

In the outer shear layer, two significant changes are worth mentioning. First, due to the strong Coandă effect in Build 2, high-order moments decrease near the edge of the shear layer as the flow is redirect towards positive $y$-locations. The predominant local acceleration effect of Build 2 produces the opposite result, increasing the skewness 


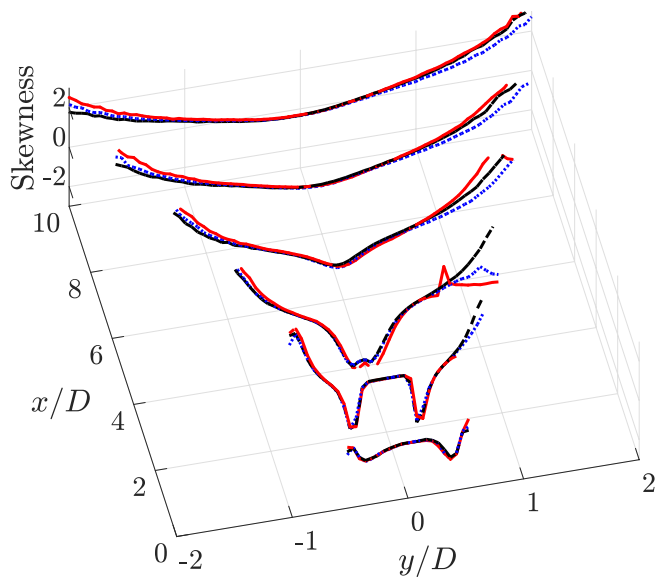

(a) Skewness radial profiles

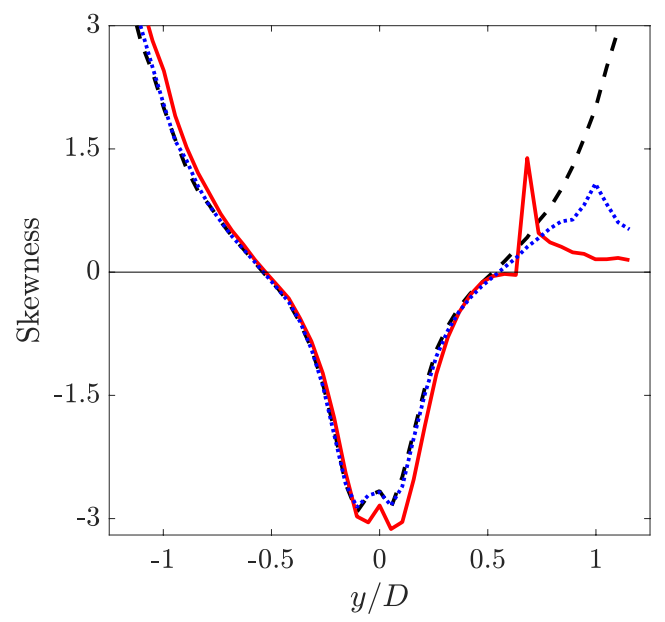

(c) Skewness at $x / D=4$

Fig. 6 Skewness and kurtosis distribution of an isolated jet and installed jet configurations. a, b Radial profiles at $x / D=2,4,6,8$ and $10 ; \mathbf{c}, \mathbf{d}$ radial profiles at axial location $x / D=4$ (note that the values of a normal distribution are indicated by a black, solid

and the kurtosis near the edge of the shear layer. Second, a sharp increase in both skewness and kurtosis occurs near the plate's trailing edge location of both installed cases. This result, once more, is consistent with the view of flow separation generating a strongly intermittent event immediately downstream of the plate trailing edge. As expected, the dynamics of the flow closed to the surface of a jet-plate configuration is complex. Results presented here suggest that the change in the jet mixing noise sources due to this interaction is highly dependent on the plate location. A similar analysis on the transverse velocity components and on the jet pressure field is expected to provide further insight and will be carried out in future campaign.

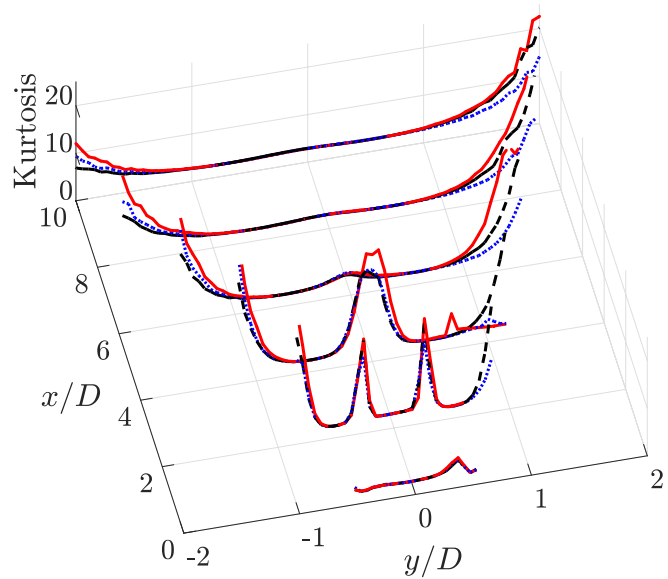

(b) Kurtosis radial profiles

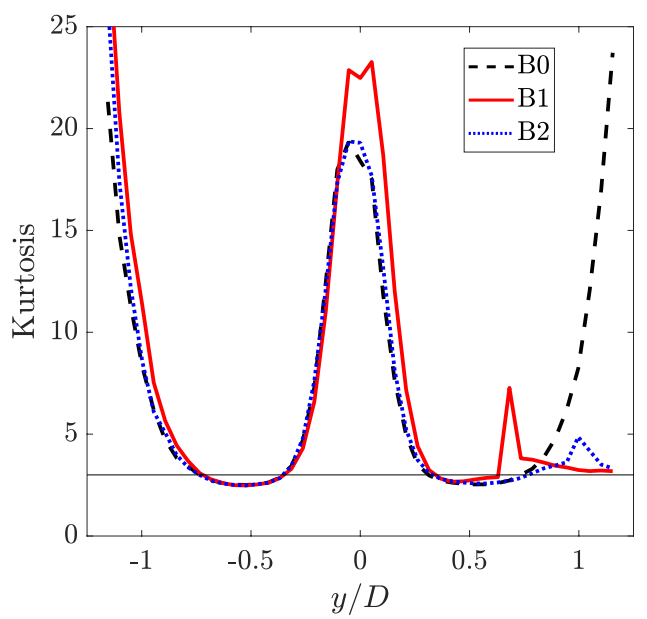

(d) Kurtosis at $x / D=4$

line). Black, dashed lines for isolated jets (B0); red, solid lines for installed $h / D=0.67, l / D=4$ jet (B1); blue, dotted lines for installed $h / D=1.00, l / D=4$ jet (B2). All results for jet exit Mach number $M=0.6$

In the next Section, the two-point statistics are surveyed and characteristic turbulence scales and eddy convection velocities are discussed.

\section{Results: turbulence characteristic scales and eddy convection velocity}

\subsection{Cross-correlation and coherence functions}

Space-time cross-correlation and coherence functions were used to calculate characteristic time scales and length scales of the jet flow field. Second-order and fourth-order 
coefficients, which are based on the velocity fluctuations and on the square of the velocity fluctuations, were calculated. In mathematical terms, the normalised second-order cross correlation function is,

$R_{11}(\boldsymbol{y}, \zeta, \tau)=\frac{\overline{u^{\prime}(\boldsymbol{y}, t) u^{\prime}(\boldsymbol{y}+\zeta, t+\tau)}}{\overline{u^{\prime}(\boldsymbol{y}) u^{\prime}(\boldsymbol{y}+\zeta)}}$.

Second-order and fourth-order joint moments were seen to produce similar trends when comparing isolated and installed jet configurations. Thus, only second-order coefficients will be displayed in the study presented below. The coherence function is the Fourier transform of the cross-correlation function defined in Eq. 1. These time and frequency domain coefficients were obtained by applying the procedure described in Sect. 2.3 to the velocity discrete time series.

Jet characteristic length- and time scales obtained from the coefficients of the joint moments described above are discussed in the next two subsections. For a more detailed discussion about the joint moments and sample results of the current database, the interested reader may refer to Proença (2018) and Proença et al. (2019).

\subsection{Characteristic length scales}

In the time domain, the integral length scale is obtained along the ordinate axis (i.e. in a fixed-frame of reference, $\tau=0$ ) of the space-time cross-correlation coefficients. Coefficients along this fixed-frame of reference are also known as space correlation coefficients. Two popular definitions of the integral length scales are (1) the integral of the area under the space correlation coefficients in the range $0 \leq \zeta \leq \infty$; and (2) the separation distance in which the fixed frame cross-correlation coefficient is equals to $1 / \mathrm{e}$. Note that, in the high turbulent kinetic energy jet region, the space correlation function can be modelled as a one-term exponential (Proença et al. 2019). Thus, the 1/e method and the classical definition of characteristic turbulence length scales are equivalent. Translating into mathematical terms, it yields,

$\mathcal{L}_{11}(t)=\zeta\left(R_{11}(\zeta, 0)=1 / \mathrm{e}\right)=\int_{0}^{\infty} R_{11}(\zeta, 0) \mathrm{d} \zeta$.

Figure 7 illustrate the decay of the space cross-correlation coefficients for an isolated and two installed cases. The axial separation distance $(\zeta(\xi, 0,0))$ is normalised by the shear layer width, $\delta_{\beta}$ measured at the reference sensor location. The reference sensor is located at $x=5 D$, on the lipline of both lower and upper shear layers. The jet Mach number is 0.6 for all cases. Isolated and upper shear layer data $(y=0.5 D)$ are illustrated by closed symbols. Open symbols illustrate the coefficients of the two installed cases on the lower shear layer.

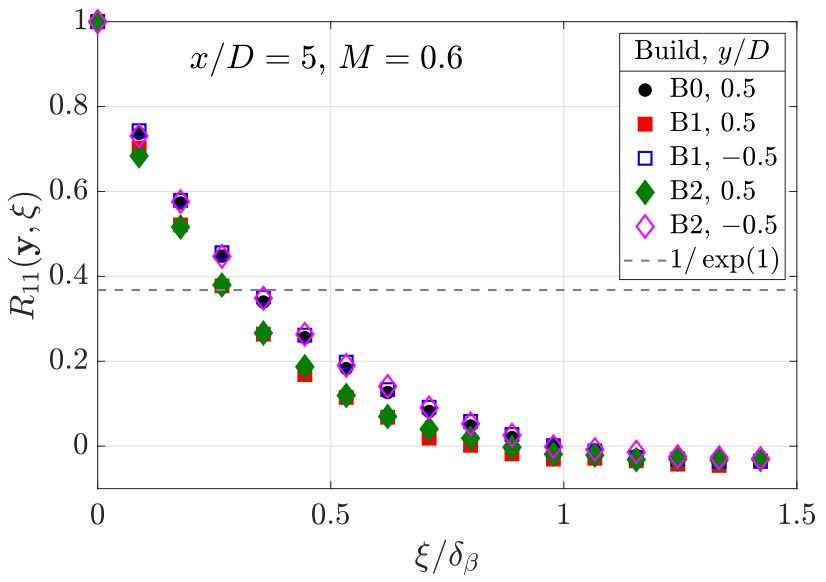

Fig. 7 Longitudinal space cross-correlation coefficients of isolated and installed configurations at $x / D=5, y / D= \pm 0.5$. Closed symbols show data measured on the upper shear layer. Open symbols measured on the lower shear layer. Jet Mach number $M=0.6$

As the isolated jet case analysed is axisymmetric, no significant differences were seen for the coefficients measured at $y=0.5 D$ and $y=-0.5 D$. Thus, only one location is shown in Fig. 7. The space correlation coefficients measured on the unshielded side of installed configurations collapse well with the isolated coefficients. On the other hand, the correlation coefficients are consistently lower for installed configurations on the shear layer close to the plate, indicating a lower characteristic length scale in those locations, as expected. Similar trends were observed when the reference sensor was located at $x / D=4$ and 8 and for other jet exit velocities ( $M=0.2$ and 0.4$)$.

The axial length scale was obtained by interpolating the separations distances of the cross-correlation coefficients closest to the 1/e value. Radial and azimuthal length scales were also calculated. All three Mach numbers studied $(M=0.2,0.4$ and 0.6$)$ produced similar results and the final length scales at each location were averaged. Table 2 summarises the values found for axial, radial and azimuthal length scales at $x / D=4$ and $8, y / D=0.5$. The consistent decrease of the characteristic length scale in the upper shear layer due to the presence of the plate is in agreement with the decrease in energy of the large-scale structures discussed in Sect. 3.2. It is a direct effect of the sudden interruption of the shear layer growth imposed by the rigid body.

It has been shown recently that the shear layer width collapses the single-point and two-point statistics of isolated jets from the nozzle exit up to at least two potential core lengths (Proença et al. 2019). This claim is also valid for axial and radial length scales of the installed configurations studied here. However, the azimuthal length scales at $x / D=4$ and $x / D=8$ of the installed configurations differ significantly. This is due to the break in the azimuthal 
Table 2 Turbulence characteristic length scales calculated from space crosscorrelation coefficients

\begin{tabular}{lllllll}
\hline Scale & Isolated & \multicolumn{3}{l}{$h=0.67 D$} & \multicolumn{3}{c}{$h=1.00 D$} \\
\hline$x / D$ & 4 & 8 & 4 & 8 & 4 & 8 \\
$\mathcal{L}_{\xi} / \delta_{\beta}$ & 0.31 & 0.31 & 0.27 & 0.29 & 0.28 & 0.29 \\
$\mathcal{L}_{\eta} / \delta_{\beta}$ & 0.19 & 0.19 & 0.16 & 0.18 & 0.18 & 0.18 \\
$\mathcal{L}_{\Delta \theta} / \delta_{\beta}$ & 0.14 & 0.15 & 0.09 & 0.13 & 0.10 & 0.13 \\
\hline
\end{tabular}

Results based on the second-order coefficients, central point on the lipline (shielded side) structures close to the plate surface and it is discussed in more detail in Sect. 4.4.

For jet mixing noise models, it is more practical to use characteristic lengths scales which are frequency dependent (Harper-Bourne 2003; Self 2004). These are obtained from the decay of the magnitude of the complex coherence function, $\gamma$. As for the cross-correlation, the complex coherence is accurately represented by a one-term exponential function. The frequency-dependent length scale is given by,

$\mathcal{L}_{11}\left(f_{i}\right)=\zeta\left(\gamma_{11}\left(\zeta, f_{i}\right)=1 / \mathrm{e}\right)=\int_{0}^{\infty} R_{11}\left(\zeta, f_{i}\right) \mathrm{d} \zeta$

where $f_{i}$ is a predetermined frequency. Based on a low Mach number jet ( $M=0.2$ ), Morris and Zaman (2010) proposed the following fit to the frequency-dependent characteristic axial length scale,

$\mathcal{L}_{\xi} / D=\left[1-\exp \left(-C_{\mathrm{s}} \mathrm{St} L_{I} / D\right)\right] / C_{\mathrm{s}} \mathrm{St}$,

where $L_{I}$ corresponds to the value of the low frequency length scales normalised by the jet nozzle diameter and $C_{\mathrm{s}}$ is a constant adjusted by the experimental results.

In jet regions of high turbulence kinetic energy, the frequency-dependent length scale follows the same trend as the PSD measured in that region: the low-frequency region is flat and the relatively high-frequency region will decay proportionally to a power of the frequency. ${ }^{2}$ It was then noted in this work that a von Kármán spectrum type of function can be used to model the frequency-dependent length scale. It is necessary to define two parameters, namely (1) the value of the largest length scale normalised by the shear layer thickness; and (2) a cut-off Strouhal number defined by the intersection of the $\mathrm{St}^{-1}$ decay of the high-frequency content and a flat line defined by the low-frequency scales. Defining these parameters, respectively, as $L_{I}$ and $\mathrm{St}_{0}$, the equation for the frequency-dependent axial length scale is written as,

$\mathcal{L}_{\xi} / \delta_{\beta}=L_{I}\left[1+\left(\mathrm{St}_{\beta} / \mathrm{St}_{0}\right)^{2}\right]^{-1 / 2}$.

Results of the measured frequency-dependent length scale are illustrated in Fig. 8. Three axial locations are shown for

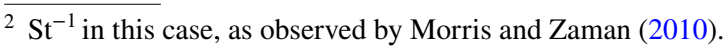

the installed builds 0,1 and 2. To obtain the parameters $L_{I}$ and $\mathrm{St}_{0}$, the experimental data were fitted by Eq. 5 using a non-linear least-square solver. The best-fits to the experimental data (symbols) are illustrated by lines. Values of $L_{I}$ and $\mathrm{St}_{0}$ derived from the best-fit are displayed in the captions of each sub-figure.

In Eq. 5 and Fig. 8, the width used to define the Strouhal number $\mathrm{St}_{\beta}$ is the shear layer width at the reference sensor location. However, scaling the jet frequency dependent lengths scales at different locations is not as straightforward as the previous properties discussed in the text. Although this fixed width collapses well the decay of the length scales at high frequencies, the cut-off frequency along the jet lipline varies proportionally to the shear layer width of the moving sensor. The amplitude of the frequency-dependent length scales at different axial locations also collapse when normalised by the time-domain length scale at the same location instead of the shear layer width. All these properties have been discussed in Proença et al. (2019).

Instead of discussing the different scaling parameters here, $L_{I}$ and $\mathrm{St}_{0}$ are used to summarise the differences in frequency-dependent length scales of the different configurations. $L_{I}$ represents the size of the largest coherent structures in the flow and $\mathrm{St}_{0}$ is the frequency cut-off. The frequency cut-off establish the dominance of either the low-frequency or the high-frequency content of the length scales.

Figure 9 displays $L_{I}$ and $\mathrm{St}_{0}$ of two installed configurations normalised by the isolated case. The solid line shows data for the plate located at $h=0.67 D$. Dashed line represents the $h=D$ configuration. Results in Fig. 9a shows that the plate consistently decrease the size of the largest structures in the flow. This effect is stronger closer to the plate trailing edge and tends to decrease as the jet mixes further downstream. This result agrees with the time domain study.

Figure $9 \mathrm{~b}$ also demonstrates a noteworthy trend. In the installed cases, the frequency cut-off is larger in comparison to an isolated jet. This suggests that there is an energy transfer from the low-frequency region to the high-frequency region. This phenomenon is also more distinct close to the plate trailing edge, decreasing in locations farther away from the plate.

In summary, the joint moments and length scales are affected when a plate is mounted in the rotational field of a jet. This suggests changes in both the coherent and incoherent 


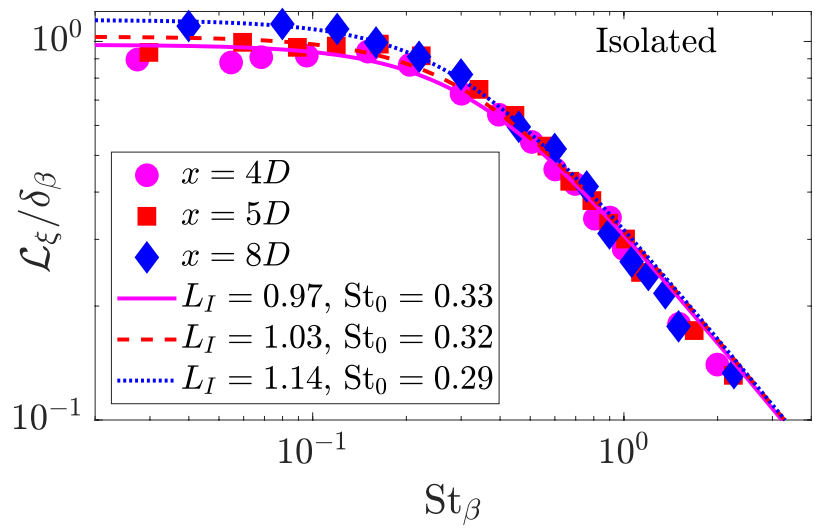

(a) Build 0

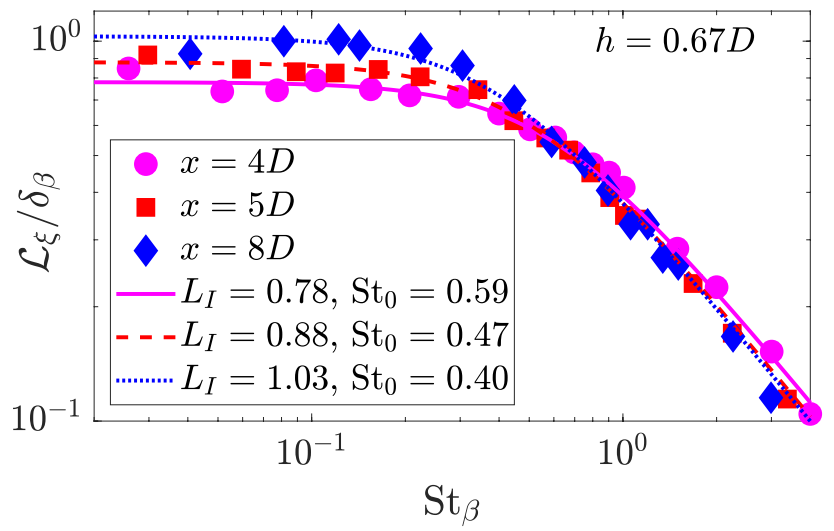

(b) Build 1

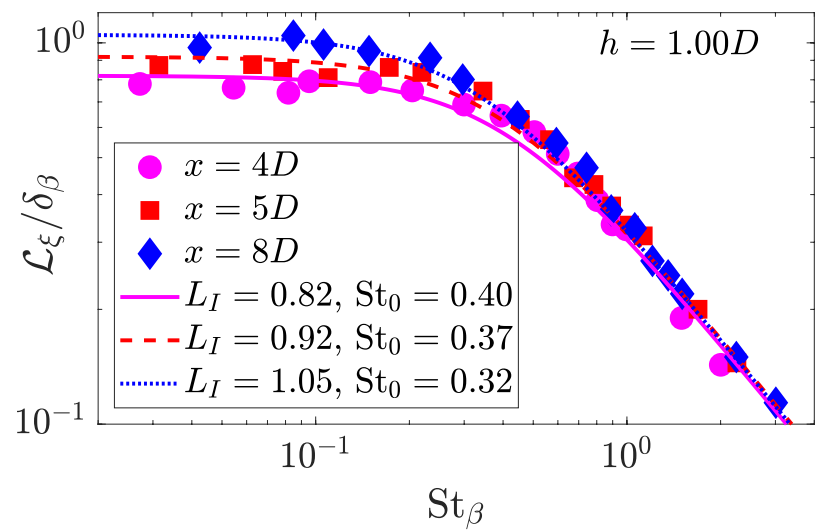

(c) Build 2

Fig. 8 Frequency-dependent length scales at $x / D=4,5$ and 8 , $y / D=0.5$. Symbols represent the experimental data and lines illustrate Eq. 5. Value of the parameters $L_{I}$ and $\mathrm{St}_{0}$ are displayed in the caption of each graph. Jet Mach number $M=0.2$

sources of jet mixing noise. Quantifying these changes would require a simultaneous evaluation of a considerable portion of the jet volume. Nonetheless, valuable information about changes in the jet statistics obtained from point-wise

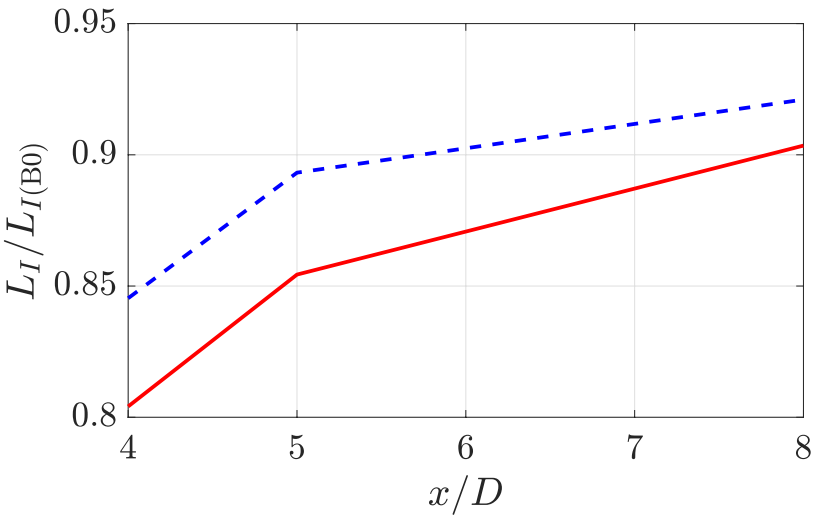

(a) $L_{I}$

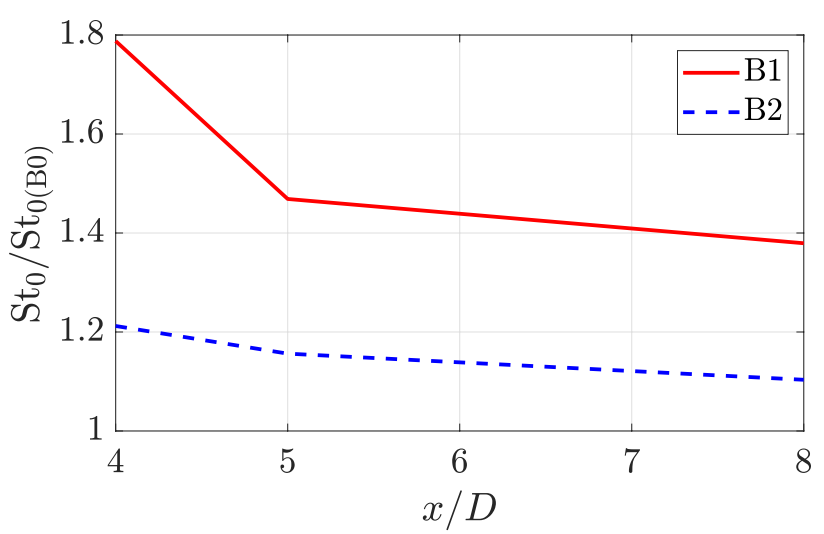

(b) $\mathrm{St}_{0}$

Fig. 9 Ratio between the $L_{I}$ and $\mathrm{St}_{0}$ parameters for installed jets and an isolated jet

measurements has been presented. No significant difference was appreciated when comparing an incompressible $M=0.2$ jet and a compressible $M=0.6$ jet.

In the next subsection, the turbulence characteristic time scale and the eddy convection velocity are interrogated to further investigate the low wave number structures in installed jets.

\subsection{Characteristic time scale and convection velocity}

The characteristic time scale studied here was defined from the decay of the space-time cross-correlation peak coefficients as the separation distance in two-point measurement increase. Using this definition, the separation of the space-time variables is only possible in the direction of the flow. Thus, for the axial velocity component, this time scale is defined in the separation direction $\zeta(\xi, 0,0)$. Using the $1 / \mathrm{e}$ method, the time scale $\mathcal{T}$ is computed as,

$\mathcal{T}_{11}=\tau\left(\max \left(R_{11}(\xi, \tau)\right)=1 / \mathrm{e}\right)$ 


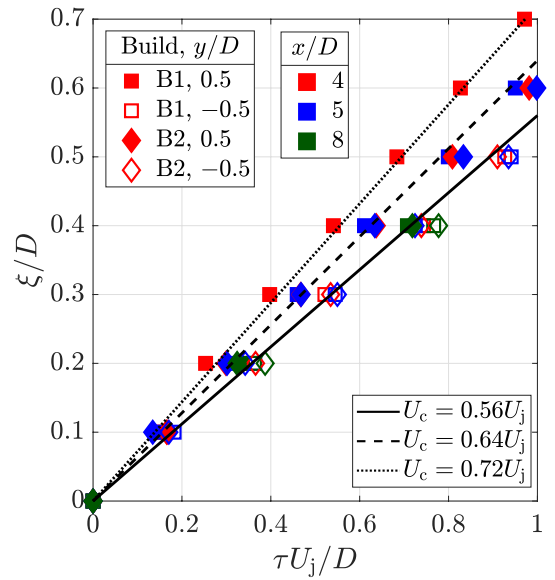

(a)

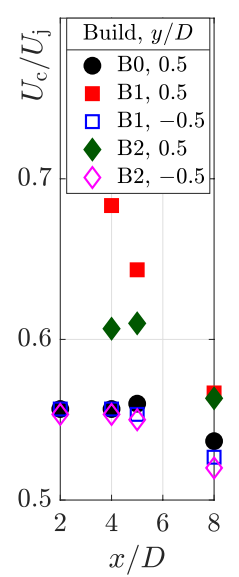

(b)
Fig. 10 a Space-time cross-correlation coefficients as function of time delay and separation distance. Symbols show data for Builds 1 and 2 at three axial locations and on $y / D= \pm-0.5$. b Eddy convection velocity computed from Eq. 7 of isolated and installed jets along the lipline

The decay of the peak coefficients defines a Gaussian-like function commonly known as the moving-frame autocorrelation. Analysis of $\mathcal{T}$ reveals similar results presented in Sect. 4.2. In the upper shear layer, the moving-frame autocorrelation of installed cases decays more rapidly in comparison to the lower shear layer and the jet isolated case. Therefore, in the shielded side, the jet time scale decreases consistently as a rigid surface is mounted closer to the jet centreline. A reduction in the characteristic time scale of these installed configurations was expected due to the local acceleration discussed in Sect. 3.1.

It is convenient to investigate the eddy convection velocity $U_{\mathrm{c}}$ at this point. It was mentioned in Sect. 3.3 that the relationship between $U_{\mathrm{c}}$ and the jet local mean velocity $\bar{U}$ depends on the local skewness. For an isolated jet, $U_{\mathrm{c}}=\bar{U}$ on the region of maximum turbulent kinetic energy, which is along the lipline for axial locations approximately up to two potential core lengths. Also, on this cylinder with radius $y=0.5 D$, the skewness is equal to zero.

The eddy convection velocity is obtained from the relationship,
$U_{\mathrm{c}(\xi, \tau)}=\left(\frac{\xi}{\tau}\right)_{\mathrm{MFA}}$,

where MFA is the abbreviation for moving-frame autocorrelation. In Fig. 10, the procedure to calculate the eddy convection velocity and results for the geometry studied are illustrated. Figure 10a shows space-time cross correlation coefficients as function of the separation distance and the time delay. Three values of eddy convection velocity are displayed by lines and the symbols show data for Builds 1 and 2 at $x / D=4,5$ and $8 ; y / D= \pm 0.5$. Figure $10 \mathrm{~b}$ exhibit the $U_{\mathrm{c}}$ value computed for isolated and installed configurations.

In summary, the difference between the $U_{\mathrm{c}}$ along the upper shear layer lipline of installed and isolated configurations is much greater than on the local mean velocity. This difference reduces further downstream of the nozzle exit. This clearly indicates the reduction in the characteristic time scale of installed jets discussed above.

Finally, an eddy convection velocity can also be estimated from the time scale obtained from single-point autocorrelation coefficients and the fixed-frame length scale presented in Sect. 4.2. In mathematical terms,

$U_{\text {c,frozen }}=\frac{\mathcal{L}_{\xi}}{\mathcal{T}_{\mathrm{FF}}}$

This is known to be a reasonable approximation only for frozen turbulence, locally isotropic flows. Table 3 displays the results computed for the fixed-frame time scale and the eddy convection velocity calculated from Eqs. 7 and 8. Axial locations $x / D=4$ and 8 are shown for Builds 0,1 and 2 .

Although the turbulence is highly non-frozen and the unsteady velocity field shows a degree of anisotropy, the convection velocity calculated from space-time crosscorrelation coefficients and in a fixed frame of reference agree well for both isolated and installed cases. In the jet region analysed, this is expected to present best results along the lipline, as this is the centre of rotation of largescale structures developing on the jet shear layer and where $u^{\prime} \sim v^{\prime} \sim w^{\prime}$ (Harper-Bourne 2003; Proença 2018).

The largest difference between the two eddy convection velocities computed, although not significant, was seen at $x / D=4$ of the closest mounted jet-plate configuration.
Table 3 Turbulence characteristic time scale, convection velocity calculated from space correlation coefficients and from assuming Taylor's hypothesis

\begin{tabular}{lllllll}
\hline & Isolated & & \multicolumn{3}{c}{$h=0.67 D$} & \multicolumn{2}{c}{$h=1.00 D$} \\
\hline$x / D$ & 4 & 8 & 4 & 8 & 4 & 8 \\
$\mathcal{T}_{\mathrm{FF}} / \delta_{\beta}$ & 0.55 & 0.55 & 0.38 & 0.52 & 0.47 & 0.52 \\
$U_{\mathrm{c}(\xi, \tau)} / U_{\mathrm{j}}$ & 0.56 & 0.54 & 0.68 & 0.57 & 0.61 & 0.56 \\
$U_{\mathrm{c} \text {,rozen }} / U_{\mathrm{j}}$ & 0.56 & 0.56 & 0.71 & 0.56 & 0.60 & 0.56 \\
\hline
\end{tabular}

Results based on the second-order coefficients, central point on the lipline (upper shear layer) 


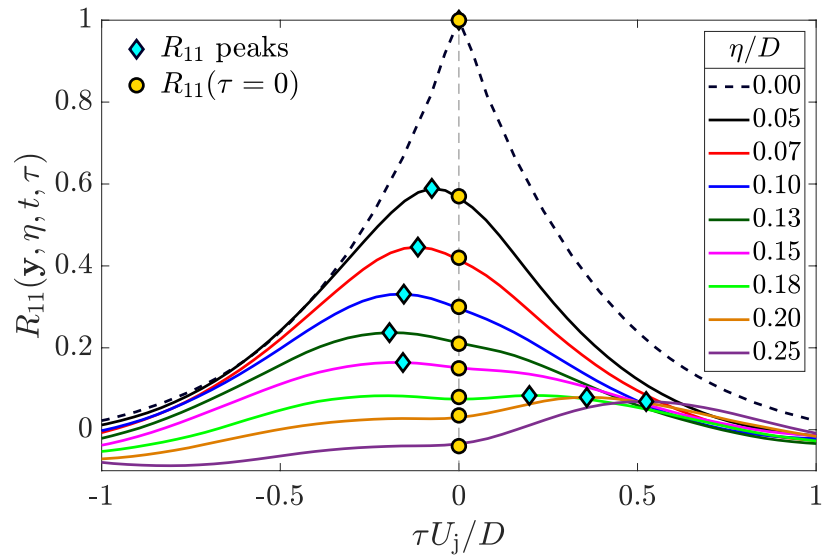

Fig. 11 Second-order space-time cross-correlation coefficients of radial separations carried out for an isolated jet. The reference sensor is located at $x / D=2, y / D=0.5$. The moving sensor traverses towards the jet centreline. Jet exit Mach number $M=0.2$

From the dataset used in this work, transverse correlation coefficients will be used to survey further the region close to the plate surface. Analysis of the behaviour of the cross-correlation coefficient of radial and azimuthal separations offer interesting suggestions about qualitatively changes in the eddy structure and it is carried out in the next subsections.

\subsection{Changes in turbulence structure}

Space-time cross-correlation coefficients obtained from radial separation traverses are illustrated in Fig. 11. Results shown are for an $M=0.2$ isolated jet. The reference sensor is located on the jet lipline, at $x / D=2$. A positive $\zeta(0, \eta, 0)$ means the second sensor moves towards the jet centreline (see Fig. 2).

As seen in Fig. 11, the cross-correlation peak coefficients represented by the cyan diamonds do not sit on the fixed frame of reference (yellow circles). This is caused by the mean velocity gradient existing between the two sensors. Put simply, the coherent part of a velocity signal first reaches the sensor where the local mean velocity is higher (in this case, the moving sensor, which is travelling towards the jet centreline). As the separation increases, there is a gradual change in the peak location. This is understood as the limit in which the two sensors lay within a correlated region of the flow, or eddy. In the example shown in Fig. 11, the peak change occurs around $0.15<\eta / D<0.18$. This is the location where the moving sensor leaves the shear layer and enters the jet potential core. When the reference sensor is located on the shear layer at an axial location downstream of the end of the potential core, the two sensors are uncorrelated when the moving sensor reached the jet centreline $(\eta>0)$ or the edge of the jet shear layer $(\eta<0)$.
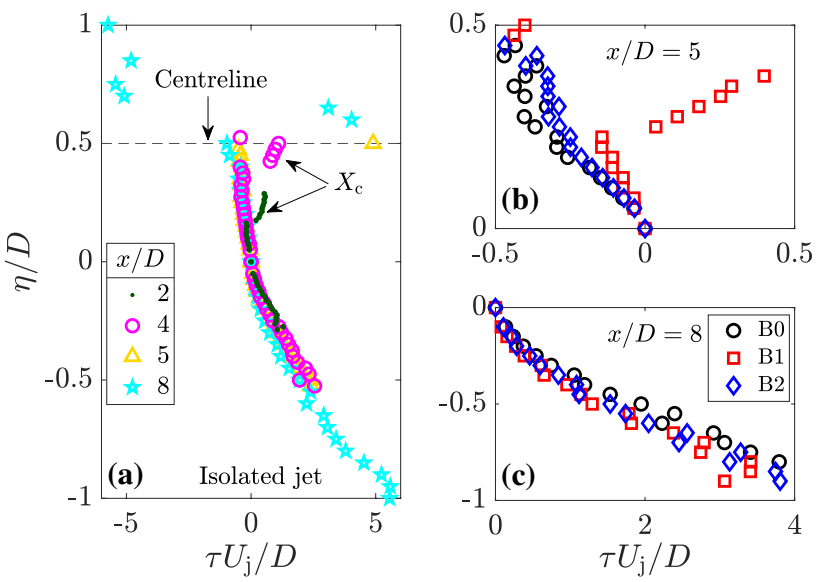

Fig. 12 Time delay of the peak coefficient obtained from two-point radial separations. a Build 0 , four axial locations; b Builds 0,1 and 2, $x / D=5 ;$ and $\mathbf{c}$ Builds 0,1 and $2, x / D=8$

Figure 12a shows the normalised time delay of the peak coefficients as function of $\eta$ for an isolated jet at four axial locations. The reference sensor is always located at $y / D=0.5$. The time delay of the peak coefficient is consistently negative for $\eta>0$, unless the moving sensor enters the jet potential core (indicated by $X_{\mathrm{c}}$ for $x / D=2$ and 4 ) or is beyond the jet centreline. As expected, the time delay of the peak coefficient is consistently positive when $\eta<0$, as the local mean velocity of the reference sensor located on the jet lipline is higher than the local mean velocity in the locations of the moving sensor which is closer to the undisturbed ambient medium.

Regarding the difference between isolated and installed jet configurations, an interesting difference occurs at $x / D=5$ (Fig. 12b). Build 1 presents a significant phase change in between the lipline and the centreline of the jet. This further advocate to the eddy break-down mechanism due to the presence of the plate. Further downstream, however, the radial peak coefficients of all configurations studied present the same behaviour (see Fig. 12c, for example), suggesting installed configurations recover the jet similarity.

Azimuthal separations also provide insights into the jet turbulence structures. For example, following the result presented in Fig. 12c, the phase of the coherence obtained from azimuthal separations is presented in Fig. 13. The reference sensor location and jet conditions are the same as in Fig. 12c. Figure 13a displays data for an isolated jet and Fig. $13 \mathrm{~b}$ for an installed configuration.

The phase of the coherence function offers information about the frequencies which contribute to the correlation coefficients. For small separation distances, signals from the two sensors are in phase for a wide range of frequencies (e.g. $\varphi<15^{\circ}$ ). As the separation increases, relatively high frequencies become uncorrelated and only the very 


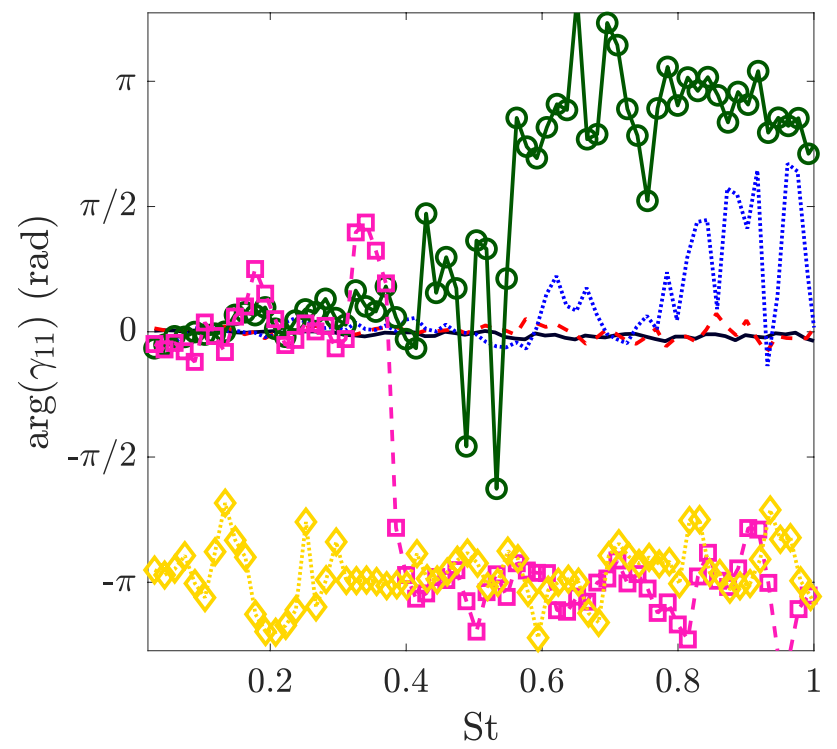

(a) Isolated jet

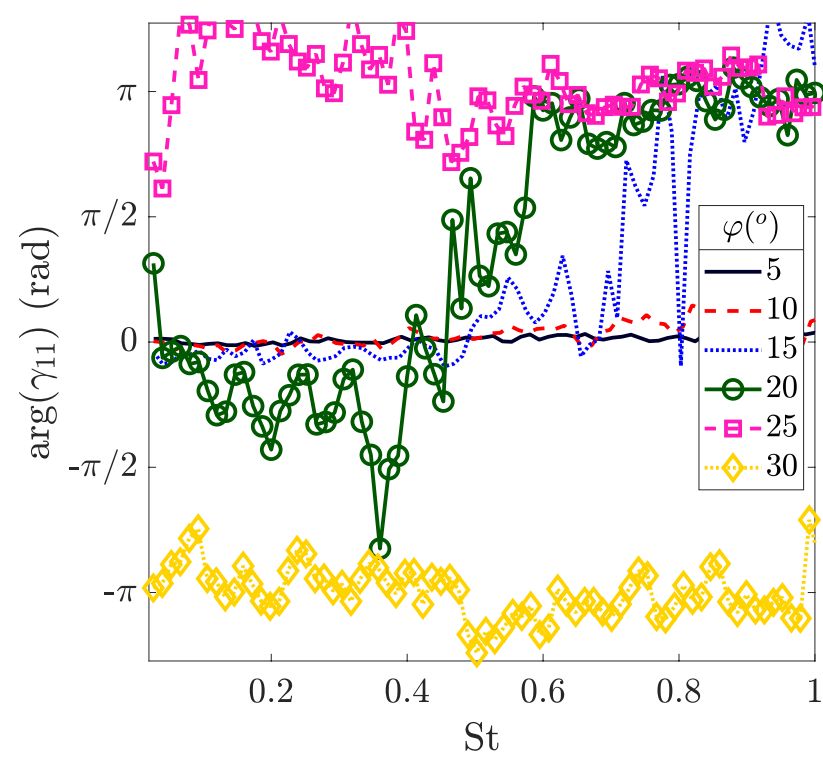

(b) $h=0.67 D, l=4 D$

Fig. 13 Azimuthal coherence phase of Builds 0 and 1. Reference sensor located at $x / D=5$, on the lipline. Jet Mach number $M=0.2$

low frequencies still contribute to the correlation between the two sensors. When the separation distance exceeds the hypothesised boundary of an eddy, the signals are in antiphase ( $\varphi=30^{\circ}$ in Fig. 13a) and the cross-correlation coefficient is zero (Proença 2018).

The presence of the plate is seen to slightly reduce the azimuthal separation distance in which the two hot-wire signals are in anti-phase. The largest difference, as for the radial separation coefficients, were seen at the location shown in Fig. 13 , at $x / D=5$. In all joint moments studied, the relative

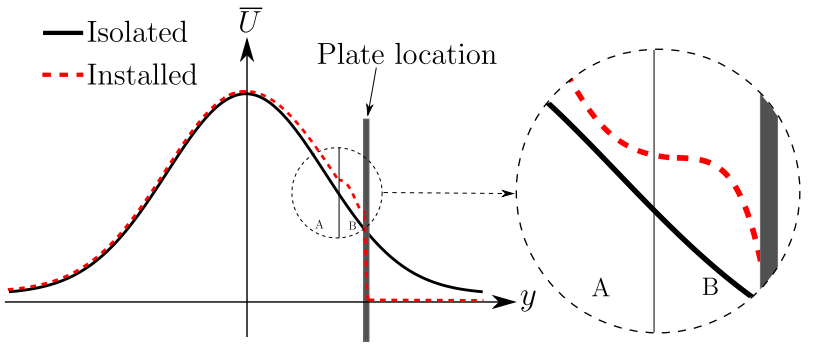

Fig. 14 Schematics of the installed jet mean velocity profile measured at $x / D=4$. Build $2(h=0.67 D)$. 'A' shows the expected mean velocity decay on the shear layer and ' $\mathrm{B}$ ' represents the velocity decay were viscous effects are non-negligible

difference between installed and isolated configurations is seen to be considerably lesser in the azimuthal direction in comparison to axial and radial separations. The evidence presented here essentially advocates that the azimuthal structure is recovered quickly downstream of the trailing edge of the solid boundary. Thus, the presence of the plate has a stronger effect on the jet statistics along the longitudinal in comparison to the transverse direction.

So far, the investigation presented has focused on the region of maximum turbulent kinetic energy on the jet shear layer. To conclude the analysis of the turbulence statistics of the installed configurations, the flow field close to the plate surface has also been studied. The main results are discussed in the next Section.

\section{Plate boundary layer analysis}

A survey has been attempt to study the boundary layer on the plate surface, downstream of the jet flow impact point. Additional single-point hot-wire traverses were performed in this region. These extra tests were aimed at identifying any significant changes to the jet development due to viscous effects acting in the jet-plate interaction region.

For the configurations analysed, this problem does not resemble either a wall-jet or a classic boundary layer problem. The flow interacting with the plate is non-uniform and reaches the plate with an oblique angle $(\beta)$. Additionally, the length in which the rotational field-plate interaction occurs is narrow, even for the closest-mounted plate $(\sim 2.5 D)$. Nonetheless, it has been seen that close to the plate trailing edge of Build 1, the jet mean velocity profile would present a change in the rate of decay near the plate. These two decay regions are illustrated in the sketch shown in Fig. 14.

Preliminary traverses along the $y$-axis were performed at axial locations $x / D=3.5,3.75$ and 3.9 and transverse locations $z / D= \pm 0.26,0.52$ and 0.79 . This would provide information about the importance of the viscous effects developing in the streamwise and spanwise directions. However, it 


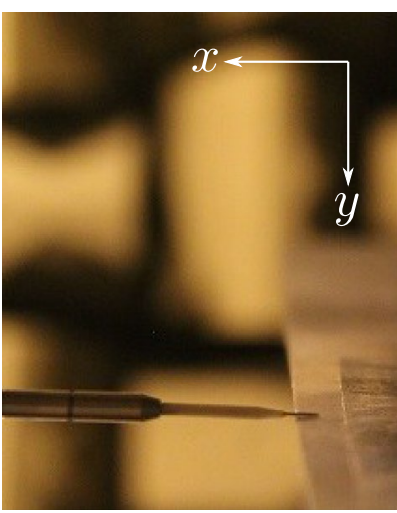

(a) $x / D=3.9$

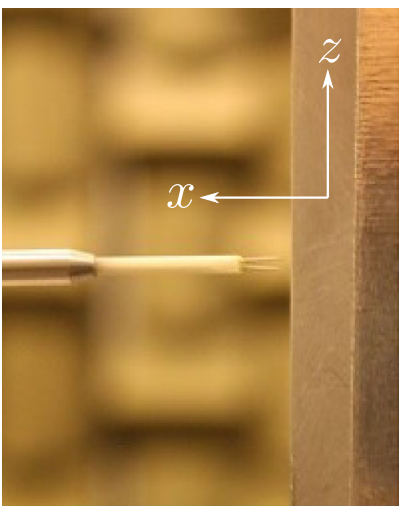

(b) $x / D=4.03$
Fig. 15 Axial location of the hot-wire during measurements upstream of and downstream of the plate trailing edge

was not possible to move the hot-wire close enough to the plate surface in the current configuration. As the plate is clamped to the nozzle, nozzle vibration with small amplitudes would propagate along the plate surface and the plate collided with the probe. Extra traverses were then performed immediately downstream of the plate trailing edge, allowing the acquisition of data in the viscous region. Figure 15 displays the hot-wire during tests in the two axial locations further investigated $(x / D=3.9$ and 4.03). All measurements were performed for a jet Mach number $M=0.6$.

Mean velocity and turbulence intensity profiles measured at three transverse locations are displayed in Fig. 16. Symbols represent data measured upstream of the plate trailing edge, whilst lines show data measured downstream of the plate trailing edge. It is seen that the mean velocity profiles measured at the two different axial locations are complementary. The transverse location closest to the jet centreline $(z / D=0.26)$ suggest the viscous effects are seen from $y / D \sim 0.66$. In other words, in that particular location and for the physical dimensions of the present experiment, the importance of viscous effects are confined in a region smaller than $0.5 \mathrm{~mm}$. Note also that, for data at $x / D=4.03$, an inflection point appears in the mean velocity and turbulence intensity profiles. This is a signature of the flow separation occurring downstream of the plate trailing edge.

The small region where viscous effects are dominant can also be inferred from the changes in the PSD of the velocity fluctuations near the plate surface. This is shown in Fig. 17. The PSD data displayed are measured immediately downstream of the plate trailing edge and perpendicular to the jet centreline. As only this location is shown, the frequency has been normalised by the jet nozzle exit diameter.

Analysis of Fig. 17 confirms that the PSD decays as $f^{-5 / 3}$ in locations $\sim 0.02 D$ away from the plate surface and then continuously change slope to a power close to -1 . Radial locations in which the PSD displays an inertial

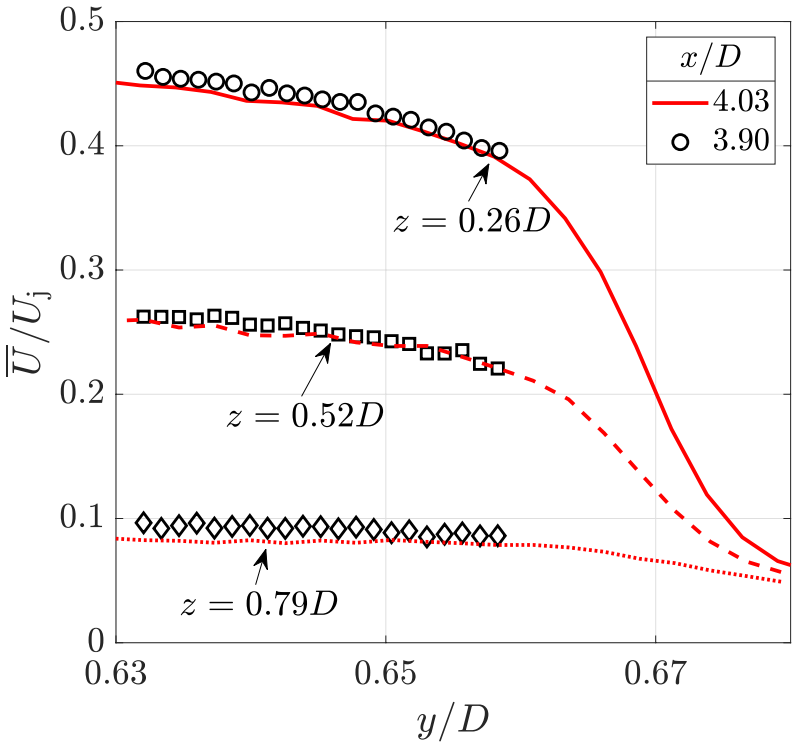

(a) mean velocity

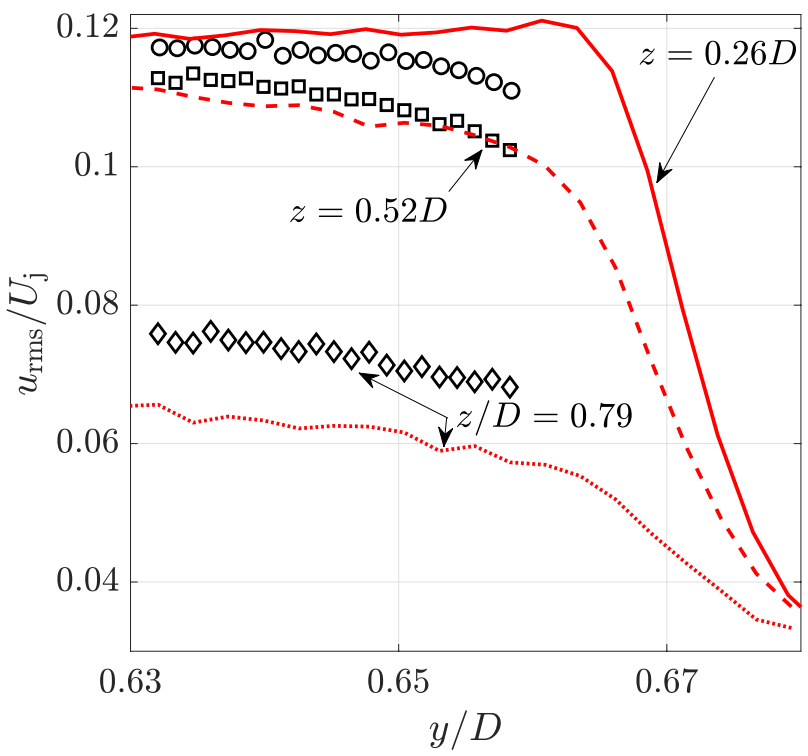

(b) turbulence intensity

Fig. 16 Jet mean velocity and turbulence intensity profiles. Three spanwise locations are shown and indicated in the graphs. Symbols represent measurements performed upstream of the plate trailing edge. Lines show data for traverses downstream of the plate trailing edge

subrange decay are dominated by the jet shear layer stresses. Therefore, viscous effects play arole only in a narrow region close to the plate trailing edge (i.e. $0 \leq \mathrm{y} / \mathrm{D}$ $\leq 0.015$ ). Thus, regarding the modelling of jet mixing noise changes due to the presence of a rigid body, boundary layer effects are negligible for high-Reynolds number applications. 


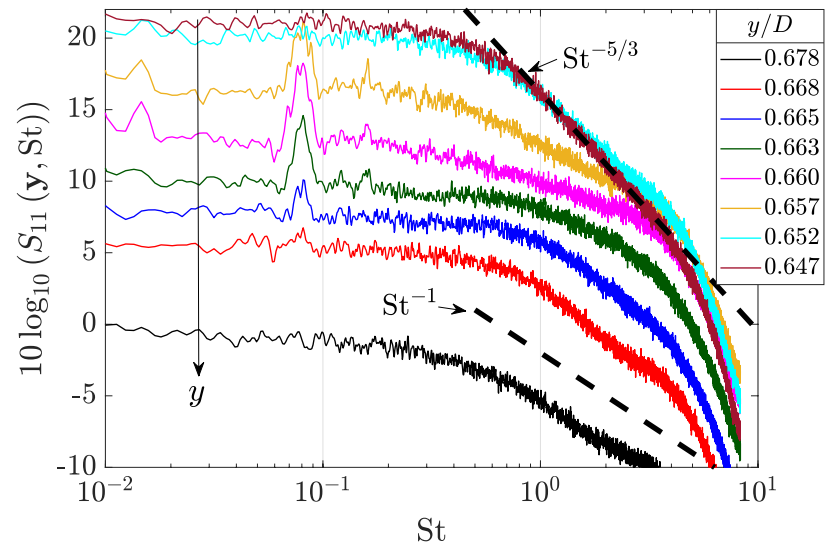

Fig. 17 PSD of the velocity fluctuations near the plate. Traverse along the $y$-axis, at $x / D=4.03$ and $z=0$

\section{Conclusions}

This paper has presented an experimental investigation into the turbulence statistics of both isolated and installed flatplate jets. Constant temperature anemometry was used to measure the unsteady axial velocity of a jet exhausting from a 38.1-mm-diameter nozzle. Single- and two-point hot-wire measurements were performed and the central and joint statistical moments were discussed in detail. Measurements were performed for several jet exit Mach numbers ranging from low-subsonic $(M=0.2)$ to high-subsonic $(M=0.8)$ velocities. The central and joint moments analysed have not shown any significant dependence on jet exit velocity for both the isolated and installed jet cases. Normalising the single-point and two-point statistics with the maximum jet exit mean velocity collapses the data both for the isolated and installed jet configurations at all Mach numbers.

One isolated and four installed jets were studied. For the two installed cases where the trailing edge of the plate was located two jet diameters downstream of the nozzle, the flow statistics were found to be similar to those observed for the isolated jet. For the other two installed configurations where the plate trailing edge was situated four jet diameters downstream of the nozzle exit, three significant changes were observed in the flow data. First, a redirection of the jet plume is observed via a Coandă effect. Second, a local acceleration of the mean flow is seen close to the plate surface. Third, a restriction of the growth of the turbulent structures.

For configurations with a four diameter chord plate, the solid body clearly restricts the amount of flow entrainment into the jet and, thus, a reduction in mixing results. The Coandă effect is observed most clearly at $h=D$, when the plate trailing edge is located almost completely outside of the jet plume. The asymmetry of the flow created by this Coandă continues to be observed further downstream of the plate trailing edge at least as far down as ten jet diameters.
The second change is clearly seen at $h=0.67 D$, where the rotational hydrodynamic field of the jet is seen to interact strongly with the rigid surface. The jet impacts the plate relatively close to the jet nozzle exit, at $\sim 1.4 D$. The flow near the plate surface undergoes a local acceleration as a direct effect of the change in momentum due to the constraining presence of the surface. In other words, the flow that would ordinarily spread radially towards the plate is redirected towards the longitudinal and spanwise directions along the plate surface. Calculation of the eddy convection velocity near the plate shows that $U_{\mathrm{c}}$ is considerably higher in the upper shear layer in comparison to the unshielded side.

The last effect studied also justifies the significant changes to the eddy convection velocity. The plate in the two $l=4 D$ builds, restricts the development of the jet and, therefore, the development of larger coherent structures in the upper shear layer. Two key consequences of this confinement were discussed, including: (1) a decrease in the low-frequency PSD energy of the unsteady velocity field, and (2) a decrease in both the characteristic length scales and time scales. The turbulence intensity is seen to decrease consistently in the upper shear layer of both installed configurations. Using the PSD, it has been seen that only the low-frequency content of the unsteady velocity changes due to the presence of the plate. The turbulence characteristic scales are also consistently lower near the plate surface. These changes have been quantified in the text.

Additionally, a qualitative approach has been used to study the cross-sectional structure of the jet. Key results from this analysis suggest that, although radial and azimuthal structures are modified near the plate surface, the length scales in the transverse direction recover self-similarity faster than the longitudinal scales. This is a good indication for jet noise prediction methods which rely on axisymmetric statistics. Finally, a survey of the unsteady velocity field near the plate surface indicates that the boundary layer developing on the plate surface is no more than $0.0125 D$ thick and it is not expected to play an important role as a noise source at frequencies of interest.

The configurations studied, although simplified in terms of geometry, were selected with the full-scale jet-wing problem in mind. This investigation is a first attempt at understanding the behaviour of the turbulence flow field of jets interacting with aircraft wing surfaces based on subtly different jet-surface positions. These results will be used to further investigate changes to the far-field jet mixing noise in closely coupled installed configurations as well as to help account for changes to the near-field jet-surface interaction noise source. Further research regarding the transverse shear stresses, realistic wing geometries, and wing angle of attack will also be carried out to complete this survey. 
Acknowledgements A. Proença would like to acknowledge financial support from the CAPES Foundation within the Brazilian Ministry of Education (Grant BEX-9333-13-4).

\section{Compliance with ethical standards}

Conflict of interest The authors declare that they have no conflict of interest.

Open Access This article is licensed under a Creative Commons Attribution 4.0 International License, which permits use, sharing, adaptation, distribution and reproduction in any medium or format, as long as you give appropriate credit to the original author(s) and the source, provide a link to the Creative Commons licence, and indicate if changes were made. The images or other third party material in this article are included in the article's Creative Commons licence, unless indicated otherwise in a credit line to the material. If material is not included in the article's Creative Commons licence and your intended use is not permitted by statutory regulation or exceeds the permitted use, you will need to obtain permission directly from the copyright holder. To view a copy of this licence, visit http://creativecommons.org/licenses/by/4.0/.

\section{References}

Batchelor GK (1982) The theory of homogeneous turbulence. Cambridge Science Classics, Cambridge

Bradshaw P (1977) Compressible turbulent shear layers. Annu Rev Fluid Mech 9(1):33-52. https://doi.org/10.1146/annur ev.fl.09.010177.000341

Brown CA, Wernet MP (2014) Jet-surface interaction test: flow measurement results. In: 20th AIAA/CEAS aeroacoustics conference, AIAA. https://doi.org/10.2514/6.2014-3198

Brown CA (2013) Jet-surface interaction test: far-field noise results. J Eng Gas Turb Power 135(7):071201. https://doi. org/10.1115/1.4023605

Bruun HH (1995) Hot-wire anemometry: principles and signal analysis. Oxford Science Publications, Oxford Univ Press, Oxford

Bychkov O, Faranosov G (2018) An experimental study and theoretical simulation of jet-wing interaction noise. Phys Acoust 64:437-452. https://doi.org/10.1017/jfm.2016.747

Cavalieri AV, Jordan P, Wolf WR, Gervais Y (2014) Scattering of wavepackets by a flat plate in the vicinity of a turbulent jet. J Sound Vib 333(24):6516-6531. https://doi.org/10.1016/j. jsv.2014.07.029

Davies POAL, Fisher MJ, Barratt MJ (1963) The characteristics of the turbulence in the mixing region on a round jet. J Fluid Mech 15(3):337-367. https://doi.org/10.1017/S0022112063000306

Dawson MF, Lawrence JLT, Self RH, Kingan MJ (2020) Validation of a jet-surface interaction noise model in flight. AIAA J 58(3):1130-1139. https://doi.org/10.2514/1.J058639

Di Marco A, Camussi R, Bernardini M, Pirozzoli S (2013) Wall pressure coherence in supersonic turbulent boundary layers. J Fluid Mech 732:445-456. https://doi.org/10.1017/jfm.2013.410

Faranosov G, Belyaev I, Kopiev V, Bychkov O (2019) Azimuthal structure of low-frequency noise of installed jet. AIAA J 57(5):18851898. https://doi.org/10.2514/1.J057476

Fisher MJ, Davies POAL (1964) Correlation measurements in a nonfrozen pattern of turbulence. J Fluid Mech 18(1):97-116. https:// doi.org/10.1017/S0022112064000076

Gauntner JW, Livingood JNB, Hrycak P (1970) Survey of literature on flow characteristics of a single turbulent jet impinging on a flat plate. In: TN D-5652, NASA
Gupta A, Choudhary H, Singh AK, Prabhakaran T, Dixit SA (2020) Scaling mean velocity in two-dimensional turbulent wall jets. $\mathbf{J}$ Fluid Mech 891:A11. https://doi.org/10.1017/jfm.2020.132

Harper-Bourne M (1999) Jet near-field noise prediction. In: 5th AIAA/ CEAS aeroacoustics conference

Harper-Bourne M (2003) Jet noise turbulence measurements. In: 9th AIAA/CEAS aeroacoustics conference and exhibit

Ho CM, Nosseir NS (1981) Dynamics of an impinging jet. part 1. the feedback phenomenon. J Fluid Mech 105:119-142. https://doi. org/10.1017/S0022112081003133

Jørgensen FE (2002) How to measure turbulence with hot-wire anemometers-a practical guide. Technical Report, Dantec Dynamics, Skovlunde, Denmark

Khavaran A (2015) Jet surface interaction - scrubbing noise in a transversely sheared mean flow. Int J Aeroacoust 14(3-4):373-412. https://doi.org/10.1260/1475-472X.14.3-4.373

Korbacher GK (1974) Aerodynamics of powered high-lift systems. Annu Rev Fluid Mech 6(1):319-358. https://doi.org/10.1146/ annurev.fl.06.010174.001535

Laurence JC (1956) Intensity, scale, and spectra of turbulence in mixing region of free subsonic jet. In: TR 1292, NACA

Lawrence J (2014) Aeroacoustic interactions of installed subsonic round jets. PhD thesis, University of Southampton, https://eprin ts.soton.ac.uk/367059/

Lawrence J, Azarpeyvand M, Self R (2011) Interaction between a flat plate and a circular subsonic jet. In: 17th AIAA/CEAS aeroacoustics conference (32nd AIAA Aeroacoustics Conference), AIAA. https://doi.org/10.2514/6.2011-2745

Lighthill MJ (1952) On sound generated aerodynamically i. general theory. Proc R Soc Lond A 211(1107):564-587. https://doi. org/10.1098/rspa.1952.0060

Lighthill MJ (1954) On sound generated aerodynamically ii. turbulence as a source of sound. Proc R Soc Lond A 222(1148):1-32. https ://doi.org/10.1098/rspa.1954.0049

Lyu B, Dowling AP, Naqavi I (2017) Prediction of installed jet noise. J Fluid Mech 811:234-268. https://doi.org/10.1017/jfm.2016.747

Madnia CK, Bernal LP (1994) Interaction of a turbulent round jet with the free surface. J Fluid Mech 261:305-332. https://doi. org/10.1017/S0022112094000352

Mancinelli M, Di Marco A, Camussi R (2017) Multivariate and conditioned statistics of velocity and wall pressure fluctuations induced by a jet interacting with a flat plate. J Fluid Mech 823:134-165. https://doi.org/10.1017/jfm.2017.307

Meloni S, Mancinelli M, Camussi R, Huber J (2020) Wall-pressure fluctuations induced by a compressible jet in installed configuration. AIAA J 10(2514/1):J058791

Monin AS, Yaglom AM (1975) Statistical fluid mechanics, volume II: mechanics of turbulence. Dover, Illinois

Morris PJ, Zaman K (2010) Velocity measurements in jets with application to noise source modeling. J Sound Vib 329(4):394-414. https://doi.org/10.1016/j.jsv.2009.09.024

Nosseir NS, Ho CM (1982) Dynamics of an impinging jet. part 2. the noise generation. J Fluid Mech 116:379-391. https://doi. org/10.1017/S0022112082000512

Piquet J (1999) Turbulent flows-models and physics. Springer, Berlin. https://doi.org/10.1007/978-3-662-03559-7

Proença A (2018) Aeroacoustics of isolated and installed jets under static and in-flight conditions. PhD thesis, University of Southampton, https://eprints.soton.ac.uk/426880/

Proença A, Lawrence J, Self R (2019) Measurements of the single-point and joint turbulence statistics of high subsonic jets using hot-wire anemometry. Exp Fluids 60(4):63. https://doi.org/10.1007/s0034 8-019-2716-3

Proença A, Lawrence J, Self R (2020) Experimental investigation into the turbulence flow field of in-flight round jets. AIAA J 10(2514/1):J059035 
Proença A, Lawrence J, Self R (2017) A survey of the turbulence statistics of a model-scale installed jet at low and moderate mach numbers. In: 23rd AIAA/CEAS aeroacoustics conference, AIAA. https://doi.org/10.2514/6.2017-3705

Roy S, Debnath K, Mazumder BS (2018) Turbulence statistics and distribution of turbulent eddies for jet flow and rigid surface interaction. Arch Mech 70(1):55-88

Self RH (2004) Jet noise prediction using the lighthill acoustic analogy. J Sound Vib 275(3-5):757-768. https://doi.org/10.1016/j. jsv.2003.06.020

Smith MJ, Miller SA (2013) The effects of surfaces on the aerodynamics and acoustics of jet flows. In: 19th AIAA/CEAS aeroacoustics conference, AIAA. https://doi.org/10.2514/6.2013-2041

Tian J, Roussinova V, Balachandar R (2012) Characteristics of a jet in the vicinity of a free surface. J Fluids Eng 134(3):031204. https ://doi.org/10.1115/1.4005739
Tritton D (1988) Physical fluid dynamics. Oxford Science Publ, Clarendon Press, Oxford

Vera J (2018) Modelling jet noise installation effects associated with close-coupled, wing-mounted, ultra high bypass ratio engines. $\mathrm{PhD}$ thesis, University of Southampton

Wang ZN, Proenca A, Lawrence J, Tucker PG, Self R (2020) Large-eddy-simulation prediction of an installed jet flow and noise with experimental validation. AIAA J 10(2514/1):J058921

Zaman KBMQ, Fagan AF, Bridges JE, Brown CA (2015) An experimental investigation of resonant interaction of a rectangular jet with a flat plate. J Fluid Mech 779:751-775. https://doi. org/10.1017/jfm.2015.453

Publisher's Note Springer Nature remains neutral with regard to jurisdictional claims in published maps and institutional affiliations. 